\title{
A New Discrete Edwards Model and a New Polymer Measure in Two Dimensions
}

\author{
Sergio Albeverio ${ }^{1}$, Xian Yin Zhou ${ }^{2, \star}$ \\ ${ }^{1}$ Institute of Mathematics, Ruhr-University Bochum, Postfach 102148, D-44780 Bochum, Germany. \\ BiBoS; SFB 237 Bochum-Essen-Düsseldorf; CERFIM, Locarno \\ ${ }^{2}$ Department of Mathematics, Beijing Normal University, Beijing 100875, P.R. China and Institute \\ of Mathematics, Ruhr-University Bochum, Postfach 102148, D-44780 Bochum, Germany
}

Received: 31 March 1995

\begin{abstract}
The new discrete Edwards models in this paper are defined in terms of the so-called restricted intersection local times of the lattice random walk in two dimensions. We study the asymptotic behaviours of these new discrete Edwards models in the superrenormalizable cases. In particular, by approximating these models we can construct new polymer measures in two dimensions which are different from the original polymer measures obtained by approximating the original discrete Edwards models. The new discrete Edwards models can be thought of as zero-component lattice $\phi^{4}$-fields with different cutoffs in the free and interacting parts.
\end{abstract}

\section{Introduction}

Let $\left\{B_{t}\right\}_{t \geqq 0}$ be the Brownian motion in $R^{d}$. The so-called polymer measure (or Edwards model) is formally defined by

$$
v_{\lambda}(d \omega)=N_{\lambda}^{-1} \exp \left(-\lambda \int_{0}^{1} \int_{0}^{1} \delta\left(B_{s}-B_{t}\right) d s d t\right) \mu(d \omega),
$$

where $\lambda \geqq 0$ is the coupling constant, $N_{\lambda}$ is the normalization constant and $\mu$ is the Wiener measure. There has been a lot of works on the existence of the polymer measure $v_{\lambda}$. For instance, Varadhan (see Appendix to [28]) first proved the existence of $v_{\lambda}$ for $d=2$, and Stoll [26] then used the nonstandard approach to give a proof for the existence of the polymer measure $v_{\lambda}$ for $d=2$. For $d=3$ and small enough $\lambda>0$, Westwater [29] first constructed the polymer measure $v_{\lambda}$. At the same time as discussing the Borel summability, Westwater [30] proved that the polymer measure $v_{\lambda}$ is also well defined for $d=3$ and all $\lambda \in[0, \infty)$. Recently, Bolthausen [7] used an alternative approach with a simple proof, inspired by the approaches presented in [10] and [13], to construct the polymer measure $v_{\lambda}$ for $d=3$ and small enough $\lambda>0$. In the following considerations we always assume that $v_{\lambda}$ for $d=3$ is the polymer measure defined by Bolthausen. For $d=4$, it

\footnotetext{
* Partly supported by AvH Foundation.
} 
was shown in [3] that a measure related to $v_{\lambda}$ exists if the coupling constant $\lambda$ is non-positive and infinitesimal.

Let $\left\{X_{n}\right\}_{n \geqq 0}$ be the simple random walk in $Z^{d}$ on the probability space $(\Omega, \mathscr{F}, P)$, and

$$
T_{m, n}=2 \cdot n^{(d-4) / 2} \sum_{i=1}^{n} \sum_{j=i+m}^{n} \delta\left(X_{i}, X_{j}\right) .
$$

As for $v_{\lambda}$ we can formally define a probability measure $\mu_{m, n, \lambda}$ on the space $C_{0}\left([0,1] \rightarrow R^{d}\right)$ by

$$
\mu_{m, n, \lambda}(d \omega)=\left(E \exp \left(-\lambda T_{m, n}\right)\right)^{-1} \exp \left(-\lambda T_{m, n}\right) d P,
$$

where $E$ is the expectation with respect to $P$ (for the precise definition of $\mu_{m, n, \lambda}$, we refer to [2]). We shall call the measure $\mu_{1, n, \lambda}$ "(original) discrete Edwards model" (see [20 or 24]). For $m_{n}>1$, the measure $\mu_{m_{n}, n, \lambda}$ has been called a modified discrete Edwards model in [2]. Heuristically, the intersection local time $\int_{0}^{1} \int_{0}^{1} \delta\left(B_{s}-B_{t}\right) d s d t$ can be approximated by $T_{1, n}$ (see [20 or 24]). Hence, it is strongly believed that the measure $v_{\lambda}$ can be approximated by $\mu_{1, n, \lambda}$. Indeed, Stoll [26] already proved this assertion for $d=2$. In fact, it was shown in [2] that $v_{\lambda}$ can also be approximated by $\mu_{m_{n}, n, \lambda}$ for $d=2$ if $\lim _{n \rightarrow \infty} m_{n} / n=0$. Moreover, [2] shows that $v_{\lambda}$ for $d=3$ can also be approximated by $\mu_{m_{n}, n, \lambda}$ if $\lim _{n \rightarrow \infty} n / m_{n}=\infty$. By means of this approximation, the polymer measure $v_{\lambda}$ for $d=3$ and arbitrary $\lambda \in[0, \infty)$ was constructed in [2] by using a similar approach as in [7]. For $d=4$, some other kinds of discrete models were discussed (see e.g. $[4,6,11$ and 19]).

For the case of a non-positive coupling constant $\lambda$, there has been also a lot of work on the model $\mu_{1, n, \lambda}$ or some other modified models (see e.g. $[3,8,9,14]$ ). In [22], Le Gall obtained some estimates on the exponential moments for the renormalized self-intersection local time of planar Brownian motion.

We remark that the measure $\mu_{m, n, \lambda}$ is only related to the intersections of the random walk $\left\{X_{n}\right\}$ with long ranges if $m$ is "not too small compared to $n$." As mentioned before, however, we know that the measure $v_{\lambda}$ for $d=2$ and 3 can be approximated by $\mu_{m_{n}, n, \lambda}$ if $m_{n}$ is "not too small compared to $n$." From this point of view we can say that the measure $v_{\lambda}$ is only related to the intersections of Brownian motion $\left\{B_{t}\right\}$ with long ranges. In this sense, the intersections of Brownian motion within a short range are ignored in the construction of the polymer measure $v_{\lambda}$ which were considered in the literature mentioned before. In this paper we will propose new discrete Edwards models defined in terms of the so-called restricted intersection local time of the simple random walk in two dimensions. These new discrete Edwards models are indeed related to the intersections of the random walk within short ranges. We will study the asymptotic behaviours of these new models and define the new polymer measures as their limits.

By the random walk representation of the lattice $\phi^{4}$-field (see $[3,12,15,17,28]$ ) we know that there is a tight connection between the intersections of random walks (or Brownian motions) and the $\phi^{4}$-field, a quantum field model. In fact, the Edwards model can be formally thought of as the zero-component $\phi^{4}$-field (see e.g. $[6,10,16])$. The discrete Edwards model $\mu_{1, n, \lambda}$ (see (1.2)), which is defined in terms of the so-called intersection local time of the lattice random walk, can be thought of as the zero-component lattice $\phi^{4}$-field with the same cutoff in the free and interacting parts. However, the new discrete Edwards model given in this paper 
(see Sect. 2 below), which is defined in terms of the restricted intersection local time of a lattice random walk, can be thought of as a zero-component lattice $\phi^{4}$-field with different cutoffs in the free and interacting parts. We will be especially interested in the two dimensional case, in which the new polymer measures constructed in the present paper can be proved to be different from the original polymer measures in two dimensions defined by Varadhan in [28] (or by Stoll in [26]). Using this fact one could hopefully obtain different continuum limits for a $\phi_{2}^{4}$-field by approximating the lattice $\phi_{2}^{4}$-field with different cutoffs in the free and interacting parts.

The remainder of this paper is organized as follows: In the next section, we introduce our new discrete Edwards models and then state the main results. In Sect. 3, we derive a reasonable estimate on the normalization constant with small coupling constants. In Sect. 4 we derive a reasonable estimate on the normalization constant for all positive and finite coupling constants. From the discussion in Sect. 5 we can see that the new measures in many cases (e.g. $\lim _{n \rightarrow \infty} n^{-1} m_{n}^{2}(\log n)^{3}=\infty$ and $\left.\left.\lim _{n \rightarrow \infty} n^{-1} m_{n}^{2}(\log n)^{5 / 2}=0\right)\right)$ are different from the original polymer measure $v_{\lambda}$ in two dimensions. In Sect. 6 , we prove that the polymer measure constructed in this paper and the original one (i.e. $v_{\lambda}$ ) are identical, if the restricted set (i.e. $Z_{m_{n}}^{2}$ below) is rather big (e.g. $\left.\lim _{n \rightarrow \infty} n^{-1} m_{n}^{2}(\log n)^{3}=0\right)$. In the Appendix we use the random walk representation to derive a formal connection between the new models and the lattice $\phi^{4}$-fields with different cutoffs in the free and interacting parts, which also explains the motivation for the present study.

\section{Models and Main Results}

As in Sect. 1, we let $\left\{X_{n}\right\}_{n \geqq 0}$ be the simple random walk in $Z^{d}$ on a probability space $(\Omega, \mathscr{F}, P)$. Let

$$
S_{m, n}= \begin{cases}2 \cdot n^{-1} m^{2} \sum_{i=1}^{n} \sum_{j=i+1}^{n} I_{\left\{X_{i}=X_{j} \in Z_{m}^{2}\right\}}, & d=2, \\ 2 \cdot n^{-1 / 2} m^{3} \sum_{i=1}^{n} \sum_{j=i+1}^{n} I_{\left\{X_{i}=X_{j} \in Z_{m}^{3}\right\}}, & d=3,\end{cases}
$$

where

$$
Z_{m}^{d}=\left\{m x: x \in Z^{d}\right\} \subset Z^{d} .
$$

It is clear that $S_{1, n}=T_{1, n}$, where $T_{1, n}$ was defined in Sect. 1. As in [5], the random variable $S_{m, n}$ (for $m>1$ ) is called the intersection local time restricted to the subset $Z_{m}^{d}$. Some renormalization results for the restricted intersection local time $S\left(m_{n}, n\right)$ were already obtained in [5]. In particular, for $d=2$ we obtain a renormalization result for $S_{m_{n}, n}$ of the type of those first obtained by Yor in [31] for the normalized intersection local time of Brownian motion in $R^{3}$, if $\lim _{n \rightarrow \infty} n^{-1} m_{n}^{2}(\log n)^{3}=\infty$ and $\lim _{n \rightarrow \infty} n^{-1} m_{n}^{2}(\log n)^{2}=0$. This renormalization result is different from that corresponding to the usual intersection local time of the simple random walk in $Z^{2}$ (see e.g. $[23,25]$ ). This gives the possibility to construct different polymer measures in two dimensions by approximation from these discrete Edwards models defined in terms of the random variable $S_{m_{n}, n}$. The main aim of this paper is in fact to construct rigorously such new polymer measures in two dimensions. We now define a formal probability measure on $C_{0}\left([0,1] \rightarrow R^{d}\right)$ in terms of the restricted intersection local time $S_{m, n}$. From now on, we always assume $d=2$ 
and $\lim \sup _{n \rightarrow \infty} n^{-1} m_{n}^{2}(\log n)^{5 / 2}<\infty$. Let

$$
\begin{gathered}
\beta=\frac{2}{\pi} \log n, \\
\gamma=m^{2} \sum_{y \in Z_{m}^{2}} \sum_{k=1}^{n} \sum_{j_{1}=1}^{k-1} \sum_{i=1}^{j_{1}} p_{i}(y) p_{j_{1}-i}(y) p_{k-j_{1}}(y),
\end{gathered}
$$

where $p_{l}(y)=P_{0}\left(X_{l}=y\right)$, and $\left\{P_{x}\right\}$ is the probability law of the random walk $\left\{X_{n}\right\}$. Let $X^{(n)} \in C_{0}\left([0,1] \rightarrow R^{2}\right)$ be defined as follows:

$$
X^{(n)}\left(n^{-1} i\right)=n^{-1 / 2} X_{i}, \quad i=0,1, \ldots, n,
$$

and $X^{(n)}$ is linear on $\left[(i-1) n^{-1}, i^{-1}\right]$ for $i=1, \ldots, n$. For $0 \leqq t_{1}<\cdots<t_{k} \leqq 1$ and $A_{1}, \ldots A_{k} \in \mathscr{B}\left(R^{2}\right)$, we set

$$
\begin{aligned}
& v_{m, n, \lambda}\left(X\left(t_{1}\right) \in A_{1}, \ldots, X\left(t_{k}\right) \in A_{k}\right) \\
& =\left(E \exp \left(-\bar{S}_{m, n}(\lambda)\right)\right)^{-1} \int_{\left\{X^{(n)}\left(t_{1}\right) \in A_{1}, \ldots, X^{(n)}\left(t_{k}\right) \in A_{k}\right\}} \exp \left(-\bar{S}_{m, n}(\lambda)\right) d P,
\end{aligned}
$$

where $E$ is the expectation with respect to $P$, and

$$
\begin{aligned}
\bar{S}_{m, n}(\lambda)= & \lambda S_{m, n}-2 \lambda n^{-1} m^{2} \sum_{k=1}^{n} \beta I_{\left\{X_{k} \in Z_{m}^{2}\right\}} \\
& +4 \lambda^{2} n^{-2} m^{2} \sum_{k=1}^{n} \gamma I_{\left\{X_{k} \in Z_{m}^{2}\right\}} .
\end{aligned}
$$

One can easily extend the cylinder measure (2.1) to a probability measure $v_{m, n, \lambda}$ on $C_{0}\left([0,1] \rightarrow R^{2}\right)$, which we write for simplicity as

$$
v_{m, n, \lambda}(d \omega)=\left(E \exp \left(-\bar{S}_{m, n}(\lambda)\right)\right)^{-1} \exp \left(-\bar{S}_{m, n}(\lambda)\right) P(d \omega)
$$

It is clear that the measures $v_{1, n, \lambda}$ and $\mu_{1, n, \lambda}$ coincide (both of them are identical to the original discrete Edwards model). For $m>1$, the measure $v_{m, n, \lambda}$ defines a new discrete Edwards model, which is different from the model $\mu_{m, n, \lambda}$ defined by (1.2). In the present paper, we first study the asymptotic behaviour of $v_{m_{n}, n, \lambda}$. One of the main results is as follows.

Theorem 2.1. If $\lim \sup _{n \rightarrow \infty} m_{n}^{2} n^{-1}(\log n)^{5 / 2}=0$, then $\left\{v_{m_{n}, n, \lambda}\right\}_{n \geqq 1}$ is tight for all $\lambda \in[0, \infty)$. Moreover, all limit measures of $\left\{v_{m_{n}, n, \lambda}\right\}$ and the original polymer measure $v_{\lambda}$ in two dimensions are different for $\lambda \in(0, \infty)$, provided $\lim _{n \rightarrow \infty} n^{-1} m_{n}^{2}$ $(\log n)^{3}=\infty$.

Remark. It seems possible that all limiting measures of $\left\{v_{m_{n}, n, \lambda}\right\}$ are singular with respect to the Wiener measure $\mu$ on $C_{0}\left([0,1] \rightarrow R^{2}\right)$ for $\lambda \in(0, \infty)$, if $\lim _{n \rightarrow \infty} n^{-1} m_{n}^{2}(\log n)^{5 / 2}=0$ and $\lim _{n \rightarrow \infty} n^{-1} m_{n}^{2}(\log n)^{3}=\infty$.

Similarly as for $v_{m, n, \lambda}(d \omega)$ we can define new probability measures on $C_{0}([0,1]$ $\rightarrow R^{2}$ ) by

$$
v_{m, n, \lambda}^{\prime}(d \omega)=\left(E \exp \left(-S_{m, n}(\lambda)\right)\right)^{-1} \exp \left(-S_{m, n}(\lambda)\right) P(d \omega)
$$


(the $S_{m, n}$ being defined at the beginning of this section) and

$$
v^{\prime \prime}{ }_{m, n, \lambda}(d \omega)=\left(E \exp \left(-\hat{S}_{m, n}(\lambda)\right)\right)^{-1} \exp \left(-\hat{S}_{m, n}(\lambda)\right) P(d \omega),
$$

where

$$
\hat{S}_{m, n}(\lambda)=\lambda S_{m, n}-2 \lambda n^{-1} m^{2} \sum_{k=1}^{n} \beta I_{\left\{X_{k} \in Z_{m}^{2}\right\}}
$$

We have the following results for $\left\{v_{m, n, \lambda}^{\prime}\right\}$ and $\left\{v^{\prime \prime}{ }_{m, n, \lambda}\right\}$.

Theorem 2.2. (i) If $\lim \sup _{n \rightarrow \infty} n^{-1} m_{n}^{2}(\log n)^{3} / \log \log n=0$, then $\left\{v^{\prime \prime}{ }_{m_{n}, n, \lambda}\right\}_{n \geqq 1}$ is tight for all $\lambda \geqq 0$, and $\left\{v_{m_{n}, n, \lambda}\right\}$ and $\left\{v^{\prime \prime} m_{n}, n, \lambda\right\}$ have the same asymptotic behaviour.

(ii) If $\lim \sup _{n \rightarrow \infty} m_{n}^{2} n^{-1}(\log n)^{3}=0$, then

$$
v_{m_{n}, n, \lambda}, \quad v_{m_{n}, n, \lambda}^{\prime}, \quad v_{m_{n}, n, \lambda}^{\prime \prime} \stackrel{\mathscr{D}}{\rightarrow} v_{\lambda}, \quad n \rightarrow \infty
$$

for all $\lambda \in[0, \infty)$, where $\mathscr{D}$ represents the weak convergence and $v_{\lambda}$ is the original polymer measure in two dimensions defined in Sect. 1.

Remark. We do not know whether or not $\left\{v_{m_{n}, n, \lambda}^{\prime}\right\}$ is still tight for some $\lambda>0$, if $\lim _{n \rightarrow \infty} n^{-1} m_{n}^{2}(\log n)^{3}=\infty$.

\section{On the Small Coupling Constants}

As in [2], we first give a reasonable estimate on the normalization constant for small coupling constants. The main result in this section is as follows.

Proposition 3.1. If $\lim \sup _{n \rightarrow \infty} m_{n}^{2} n^{-1}(\log n)^{5 / 2}<\infty$, then there are constants $\lambda_{0}>0, c_{1} \in(0,1)$ such that

$$
c_{1} \leqq E \exp \left(-\bar{S}_{m_{n}, n}(\lambda)\right) \leqq c_{1}^{-1}, \quad \forall \lambda \in\left[0, \lambda_{0}\right] .
$$

In particular, if $\limsup _{n \rightarrow \infty} m_{n}^{2} n^{-1}(\log n)^{3}<\infty$, then there is a constant $c_{2} \in$ $(0, \infty)$ such that $\gamma=c_{2} n+O(1)$ for $i=1, \ldots, n$.

The idea to prove Proposition 3.1 is basically from the proof of [2, Theorem 3.1]. However, some arguments here are different from those given in the proof of [2, Theorem 3.1]. We prefer to give here a slightly detailed proof of Proposition 3.1. From the proof given below one can also see why we have to assume in Proposition 3.1 that

$$
\limsup _{n \rightarrow \infty} m_{n}^{2} n^{-1}(\log n)^{5 / 2}<\infty
$$

As in [2], we set

$$
\begin{gathered}
p_{n}(x)(=: p(n, x))=P\left(X_{n}=x\right) \\
\bar{p}_{n}(x)(=: \bar{p}(n, x))=2\left(\frac{d}{2 \pi n}\right)^{d / 2} \exp \left(-\frac{d|x|^{2}}{2 n}\right) .
\end{gathered}
$$


For $m=m_{n}$, we set

$$
\begin{aligned}
& \beta_{j}(=: \beta(m, n))=\sum_{j=1}^{n} p_{j}(0)=\beta+O(1), \\
& \gamma_{j}(=: \gamma(m, n))=\gamma \\
& J_{i_{1}, j_{1} ; i_{2}, j_{2}}=\lambda m^{2} n^{-1} \sum_{i=i_{1}}^{j_{1}} \sum_{j=i_{2}}^{j_{2}} \sum_{x \in Z_{m}^{2}} I_{\left\{X_{i}=X_{j}=x\right\}}, \\
& J_{k, l}^{m, \lambda}=\lambda m^{2} n^{-1} \sum_{i=k}^{l} \sum_{j=i+1}^{l} \sum_{x \in Z_{m}^{2}} I_{\left\{X_{i}=X_{j}=x\right\}}, \\
& \bar{J}_{k, l}^{m, \lambda}=J_{k, l}^{m, \lambda}-m^{2}\left(\lambda n^{-1} \sum_{i=k}^{l} \beta_{i}-\lambda^{2} n^{-2} \sum_{i=k}^{l} \gamma_{i}\right) I_{\left\{X_{i} \in Z_{m}^{2}\right\}}, \\
& g_{k, l}^{m, \lambda}(x)=E \exp \left(-\bar{J}_{k, l}^{m, \lambda}\right) \delta\left(X_{l}, x\right), \\
& g_{l}^{m, \lambda}(x)=g_{1, l}^{m, \lambda}(x) .
\end{aligned}
$$

For notational convenience, we will henceforth drop $m$ and $\lambda$ from the above notations. We further set

$$
\begin{aligned}
\mathscr{A}_{l}^{(1)}= & \sum_{k=1}^{l} E\left[\operatorname { e x p } ( - \overline { J } _ { k + 1 , l } ) \left(\lambda n^{-1} m^{2} \sum_{j=k+1}^{l} \sum_{y \in Z_{m}^{2}} I_{\left\{X_{k}=X_{j}=y\right\}}\right.\right. \\
& \left.\left.-m^{2}\left(\lambda n^{-1} \beta_{k}-\lambda^{2} n^{-2} \gamma_{k}\right) I_{\left\{X_{k} \in Z_{m}^{2}\right\}}\right) \delta\left(X_{l}, x\right)\right] \\
\mathscr{A}_{l}^{(2)}= & \sum_{k=1}^{l} E\left[\operatorname { e x p } ( - \overline { J } _ { k + 1 , l } ) \left(1-\exp \left(-\bar{J}_{k, l}+\bar{J}_{k+1, l}\right)\right.\right. \\
& \left.\left.-\left(\bar{J}_{k, l}-\bar{J}_{k+1, l}\right)\right) \delta\left(X_{l}, x\right)\right] .
\end{aligned}
$$

Then we have

Let

$$
p(l, x)-g_{l}(x)=\mathscr{A}_{l}^{(1)}+\mathscr{A}_{l}^{(2)} .
$$

$$
\begin{aligned}
A_{l}^{(1)}= & \lambda n^{-1} m^{2} \sum_{k=1}^{l} \sum_{y \in Z_{m}^{2}}\left[\sum_{j=k+1}^{l} p_{k}(y) g_{j-k}(0) g_{l-j}(x-y)\right. \\
& \left.-\beta_{k} p_{k}(y) g_{j-k}(x-y)\right], \\
A_{l}^{(2)}= & \lambda^{2} n^{-2} m^{2} \sum_{k=1}^{l} \sum_{y \in Z_{m}^{2}}\left[\gamma_{k} p_{k}(y) g_{l-k}(x-y)\right. \\
& -\sum_{j_{1}=k+1}^{l} \sum_{i=k+1}^{j_{1}} \sum_{j=j_{1}+1}^{l} m^{2} \sum_{z \in Z_{m}^{2}} p_{k}(y) g_{i-k}(z-y) \\
& \left.\times g_{j_{1}-i}(z-y) g_{j-j_{1}}(z-y) g_{l-j}(x-z)\right],
\end{aligned}
$$




$$
\begin{aligned}
A_{l}^{(3)}= & \lambda n^{-1} m^{2} \sum_{k=1}^{l} \sum_{j=k+1}^{l} \sum_{y \in Z_{m}^{2}} E\left[I_{\left\{X_{k}=X_{j}=y\right\}}\right. \\
& \left.\times \exp \left(-\bar{J}_{k+1, j}-\bar{J}_{j+1, l}\right) J_{k+1, j ; j+1, l}^{2} \delta\left(X_{l}, x\right)\right] \\
& +\lambda^{2} n^{-2} m^{4} \sum_{k=1}^{l} \sum_{j_{1}=k+1}^{l} \sum_{i=k+1}^{j_{1}} \sum_{j=j_{1}+1}^{l} \sum_{y, z \in Z_{m}^{2}} E\left[I_{\left\{X_{k}=X_{j_{1}}=y\right\}}\right. \\
& \times I_{\left\{X_{i}=X_{j}=z\right\}} \exp \left(-\bar{J}_{k+1, i}-\bar{J}_{i+1, j_{1}}-\bar{J}_{j_{1}+1, j}-\bar{J}_{j+1, l}\right) \\
& \left.\times\left(J_{k+1, i ; i+1, j_{1}}+J_{i+1, j_{1} ; j_{1}+1, j}+J_{j_{1}+1, j ; j+1, l}\right)^{2} \delta\left(X_{l}, x\right)\right] .
\end{aligned}
$$

In general, by definition we know that $g_{l}(x) \neq g_{k, k+l-1}(x)$ for $k \geqq 2$ and $x \notin Z_{m}^{2}$. However, we can see that the conditional distribution of $\bar{J}_{k, k+l-1}$ given $\left\{X_{k-1} \in Z_{m}^{2}\right\}$ is the same as that of $\bar{J}_{1, l}$. Using this fact, as in [2 or 7] we can show that

$$
A_{l}^{(1)}+A_{l}^{(2)} \leqq \mathscr{A}_{l}^{(1)} \leqq A_{l}^{(1)}+A_{l}^{(2)}+O(1) A_{l}^{(3)}
$$

We introduce the following notation:

$$
\begin{aligned}
K(m, n)= & \sup _{1 \leqq k \leqq n} \sup _{x \in Z^{2}} \frac{\left|g_{k}(x)-p(k, x)\right|}{\mathscr{L}(k) \bar{p}(4 k, x)} \\
& \vee \max _{1 \leqq i \leqq n} \frac{\left|\sum_{j=1}^{i}\left(p_{j}(0)-g_{j}(0)\right)\right|}{\left((\log (i+1))^{-1 / 2} \log (n / i+1)\right) \vee 1},
\end{aligned}
$$

where $\mathscr{L}(k)=\frac{k}{n} \log (n / k+1)+(\log n)^{-1 / 2}$. For notational convenience, we write shortly $K$ for $K(m, n)$. Let $\phi(x)$ be a generic polynomial in $x$ with nonnegative coefficients, which might be different from line to line. We begin with several lemmas.

Lemma 3.2. There is a constant $c_{3} \in(0, \infty)$ such that

$$
m^{2} \sum_{y \in Z_{m}^{2}} \bar{p}_{4 k}(y) \bar{p}_{4 l}(x-y) \leqq c_{3}\left(1+m^{2} \frac{k+l}{k l}\right) \bar{p}(4(k+l), x)
$$

for all $k, l \geqq 1$ and $x \in R^{2}$.

Proof. It is easy to show that

$$
\begin{aligned}
& m^{2} \sum_{y \in Z_{m}^{2}} \bar{p}_{4 k}(y) \bar{p}_{4 l}(x-y) \leqq O(1) m^{2} \sum_{y \in Z_{m}^{2}} \frac{1}{k l} \exp \left(-\frac{|y|^{2}}{4 k}-\frac{|x-y|^{2}}{4 l}\right) \\
& \leqq \\
& \quad O(1) m^{2} \frac{1}{k l} \exp \left(-\frac{|x|^{2}}{4(k+l)}\right) \\
& +O(1) m^{2} \sum_{\substack{y \in Z_{m}^{2} \\
|y-k x /(k+l)| \geqq m / 2}} \frac{1}{k l} \exp \left(-\frac{(k+l)|y-k x /(k+l)|^{2}}{4 k l}-\frac{|x|^{2}}{4(k+l)}\right)
\end{aligned}
$$




$$
\begin{aligned}
& \leqq O(1) m^{2} \frac{k+l}{k l} \bar{p}(4(k+l), x)+O(1) \sum_{y \in Z^{2}} \frac{1}{k l} \exp \left(-\frac{(k+l)|y|^{2}}{16 k l}-\frac{|x|^{2}}{4(k+l)}\right) \\
& \leqq O(1)\left(1+m^{2} \frac{k+l}{k l}\right) \bar{p}(4(k+l), x),
\end{aligned}
$$

which implies the desired result.

Lemma 3.3. The following holds for all $x \in Z^{2}$ :

$$
\left|A_{l}^{(1)}\right| \leqq \lambda \phi(K)\left[\frac{l}{n} \log \left(\frac{n}{l}+1\right)+n^{-1} m^{2} \log l \log n\right] \bar{p}(4 l, x) .
$$

Proof. As in [2] we set

$$
\begin{aligned}
I_{1} & =\lambda n^{-1} m^{2} \sum_{k=1}^{l} \sum_{j=k+1}^{l} \sum_{y \in Z_{m}^{2}} p_{j}(y) g_{l-j}(x-y)\left(g_{j-k}(0)-p_{j-k}(0)\right), \\
I_{2} & =\lambda n^{-1} m^{2} \sum_{k=1}^{l} \sum_{j=k+1}^{l} \sum_{y \in Z_{m}^{2}} g_{j-k}(0)\left(p_{k}(y)-p_{j}(y)\right) g_{l-j}(x-y), \\
I_{3}= & \lambda n^{-1} m^{2} \sum_{k=1}^{l} \sum_{y \in Z_{m}^{2}}^{l}\left(\sum_{j=k+1}^{l} p_{j-k}(0) p_{j}(y) g_{l-j}(x-y)\right. \\
& \left.-\beta_{k} p_{k}(y) g_{l-k}(x-y)\right) .
\end{aligned}
$$

It is easy to show that

$$
A_{l}^{(1)}=I_{1}+I_{2}+I_{3} .
$$

As in [5] we set for $x=\left(x_{1}, x_{2}\right)$,

$$
S_{n}(x)=\left\{y=\left(y_{1}, y_{2}\right) \in Z^{2}: x_{i}-m / 2 \leqq y_{i}<x_{i}+m / 2, i=1,2\right\},
$$

and let $\eta(x)$ be the point $y$ in $Z_{m}^{2}$ such that $x \in S_{n}(y)$ (this will be used in Sect. 6).

We first consider $I_{1}$. By Lemma 3.2 we can show that $\left|I_{1}\right|$ is less than

$$
\begin{aligned}
& \lambda n^{-1} m^{2} \sum_{j=2}^{l} \sum_{y \in Z_{m}^{2}} p_{j}(y) g_{l-j}(x-y)\left(\sum_{k=1}^{j-1}\left(g_{j-k}(0)-p_{j-k}(0)\right)\right) \\
& \leqq \lambda \phi(K) n^{-1} m^{2} \sum_{j=2}^{l} \sum_{y \in Z_{m}^{2}} p_{j}(y) g_{l-j}(x-y)\left((\log j)^{-1 / 2} \log (n / j+1)\right) \vee 1 \\
& \leqq \lambda \phi(K) n^{-1} m^{2} \sum_{j=2}^{l} \sum_{y \in Z_{m}^{2}} \bar{p}_{4 j}(y) \bar{p}_{4(l-j)}(x-y)\left((\log j)^{-1 / 2} \log (n / j+1)\right) \vee 1 \\
& \leqq \lambda \phi(K) n^{-1} \sum_{j=2}^{l}\left(1+m^{2} \frac{l}{j(l-j)}\right)\left((\log j)^{-1 / 2} \log (n / j+1)\right) \vee 1 \bar{p}(4 l, x)
\end{aligned}
$$




$$
\begin{aligned}
\leqq & \lambda \phi(K)\left(n^{-1} l \log (n / l+1)(\log (l+1))^{-1 / 2}+n^{-1} m^{2}\left((\log l)^{3 / 2}\right.\right. \\
& \left.\left.+\log (n / l+1)(\log (l+1))^{1 / 2}\right)\right) \bar{p}(4 l, x) \\
\leqq & \left.\lambda \phi(K)\left(n^{-1} l \log (n / l+1) \log (l+1)\right)^{-1 / 2}+(\log n)^{-1}\right) \bar{p}(4 l, x),
\end{aligned}
$$

if $n^{-1} m^{2} \leqq O(1)(\log n)^{-5 / 2}$.

By a similar argument as for $I_{2}$ given in the proof of [2, Lemma 4.2] we can show that

$$
\left|I_{2}\right| \leqq \lambda \phi(K)\left(n^{-1} m^{2} \log (l+1)+n^{-1} l\right) \bar{p}(4 l, x) .
$$

We finally consider $I_{3}$. By the definition of $\beta_{j}$ we know that

$$
\left|I_{3}\right| \leqq \lambda n^{-1} \sum_{j=1}^{l} \phi(K) \log (n / j+1) m^{2} \sum_{y \in Z_{m}^{2}} p_{j}(y) g_{l-j}(x-y)
$$

Thus, by the same argument as for $I_{1}$ given before we can show that $\left|I_{3}\right|$ is less than

$$
\begin{aligned}
& \lambda n^{-1} \phi(K) \sum_{j=1} l\left(1+m^{2} \frac{1}{j(l-j+1)}\right) \log (n / j+1) \bar{p}(4 l, x) \\
& \leqq \lambda \phi(K)\left(n^{-1} m^{2} \log (l+1)(\log (n / l+1)+\log \log (l+1))+\frac{l}{n} \log (n / l+1)\right) \bar{p}(4 l, x) \\
& \leqq \lambda \phi(K)\left((\log n)^{-1 / 2}+l / n \log (n / l+1)\right) \bar{p}(4 l, x),
\end{aligned}
$$

if $n^{-1} m^{2} \leqq O(1)(\log n)^{-5 / 2}$. Combining the above estimates we get the desired result.

Lemma 3.4. The following holds for all $x \in Z^{2}$ :

$$
\left|A_{l}^{(2)}\right| \leqq \lambda^{2} \phi(K)\left(n^{-1} l+(\log n)^{-1}\right) \bar{p}(4 l, x) .
$$

Proof. As in the proof of [2, Lemma 4.3] we set

$$
\begin{aligned}
B_{1}= & \lambda^{2} n^{-2} \sum_{k=1}^{l} \sum_{j_{1}=k+1}^{l} \sum_{i=k+1}^{j_{1}} \sum_{j=j_{1}+1}^{l} m^{4} \sum_{y, z \in Z_{m}^{2}} p_{k}(y)\left(p_{i-k}(z-y) p_{j_{1}-i}(z-y)\right. \\
& \left.\times p_{j-j_{1}}(z-y)-g_{i-k}(z-y) g_{j_{1}-i}(z-y) g_{j-j_{1}}(z-y)\right) g_{l-j}(x-z), \\
B_{2}= & \lambda^{2} n^{-2} \sum_{k=1}^{l}\left[\gamma_{k} m^{2} \sum_{y \in Z_{m}^{2}} p_{k}(y) g_{l-k}(x-y)-m^{4} \sum_{j_{1}=k+1}^{l} \sum_{i=k+1}^{j_{1}} \sum_{j=j_{1}+1}^{l}\right. \\
& \left.\times \sum_{y, z \in Z_{m}^{2}} p_{k}(y) p_{i-k}(z-y) p_{j_{1}-i}(z-y) p_{j-j_{1}}(z-y) g_{l-j}(x-z)\right] .
\end{aligned}
$$


Then, we have

$$
A_{l}^{(2)}=B_{1}+B_{2}
$$

and that $\left|B_{1}\right|$ is less than

$$
\begin{aligned}
& \lambda^{2} n^{-2} \phi(K) \sum_{\substack{a, b, c \geqq 0 \\
a \vee b \vee c \geqq 1}} \sum_{k=1}^{l} \sum_{j_{1}=k+1}^{l} \sum_{i=k+1}^{j_{1}} \sum_{j=j_{1}+1}^{l} m^{4} \sum_{y, z \in Z_{m}^{2}}(\mathscr{L}(i-k))^{a}\left(\mathscr{L}\left(j_{1}-i\right)\right)^{b} \\
& \quad \times\left(\mathscr{L}\left(j-j_{1}\right)\right)^{c} p_{k}(y) \bar{p}_{4(i-k)}(z-y) \bar{p}_{4\left(j_{1}-i\right)}(z-y) \bar{p}_{4\left(j-j_{1}\right)}(z-y) \bar{p}_{4(l-j)}(x-z) .
\end{aligned}
$$

Let

$$
\begin{gathered}
\tau=(i-k)\left(j_{1}-i+1\right)+(i-k)\left(j-j_{1}\right)+\left(j-j_{1}\right)\left(j_{1}-i+1\right), \\
\sigma=\frac{(i-k)\left(j_{1}-i+1\right)\left(j-j_{1}\right)}{\tau} .
\end{gathered}
$$

It is clear that $\sigma \leqq j-k$.

By Lemma 3.2 we can show that

$$
\begin{aligned}
& \leqq n^{-2} \sum_{k=1}^{l} \sum_{j_{1}=k+1}^{l} \sum_{i=k+1}^{j_{1}} \sum_{j=j_{1}+1}^{l} m^{4} \sum_{y, z \in Z_{m}^{2}}(\log n)^{-1 / 2} \\
& \times p_{k}(y) \bar{p}_{4(i-k)}(z-y) \bar{p}_{4\left(j_{1}-i\right)}(z-y) \bar{p}_{4\left(j-j_{1}\right)}(z-y) \bar{p}_{4(l-j)}(x-z) \\
& \leqq n^{-2} m^{4} \sum_{k=1}^{l} \sum_{j_{1}=k+1}^{l} \sum_{i=k+1}^{j_{1}} \sum_{j=j_{1}+1}^{l}(\log n)^{-1 / 2} \\
& \times \sum_{y \in Z_{m}^{2}} p_{k}(y) \tau^{-1} \sum_{z \in Z_{m}^{2}} \bar{p}_{4 \sigma}(z-y) \bar{p}_{4(l-j)}(x-z) \\
& \leqq O(1) n^{-2} m^{2} \sum_{k=1}^{l} \sum_{j_{1}=k+1}^{l} \sum_{i=k+1}^{j_{1}} \sum_{j=j_{1}+1}^{l}(\log n)^{-1 / 2} \sum_{y \in Z_{m}^{2}} p_{k}(y) \\
& \times \tau^{-1}\left(1+m^{2} \frac{\sigma+l-j}{\sigma(l-j+1)}\right) \bar{p}(4(\sigma+l-j), x-y) \\
& \leqq O(1) n^{-2} \sum_{k=1}^{l} \sum_{j_{1}=k+1}^{l} \sum_{i=k+1}^{j_{1}} \sum_{j=j_{1}+1}^{l}(\log n)^{-1 / 2} \tau^{-1} \frac{l}{k+\sigma+l-j} \\
& \times\left(1+m^{2} \frac{\sigma+l-j}{\sigma(l-j+1)}\right)\left(1+m^{2} \frac{k+\sigma+l-j}{k(\sigma+l-j+1)}\right) \bar{p}(4 l, x) \\
& \leqq O(1)\left(\frac{l^{2}}{n^{2}}(\log n)^{-1 / 2}+\frac{m^{2}}{n} \frac{l}{n}(\log n)^{5 / 2}+\frac{m^{4}}{n^{2}}(\log n)^{4-1 / 2}\right) \bar{p}(4 l, x) \\
& \leqq O(1)\left(n^{-1} l+(\log n)^{-3 / 2}\right) \bar{p}(4 l, x),
\end{aligned}
$$

if $n^{-1} m^{2} \leqq O(1)(\log n)^{-5 / 2}$, where we have used the basic inequality: $x y \leqq$ $\frac{1}{2}\left(x^{2}+y^{2}\right)$. 
Let $\tilde{\mathscr{L}}(k)=\frac{k}{n} \log (n / k+1)$. As in the proof of [2, Lemma 4.2] we can show that if $a \vee b \vee c \geqq 1$,

$$
\begin{aligned}
& n^{-2} \sum_{k=1}^{l} \sum_{j_{1}=k+1}^{l} \sum_{i=k+1}^{j_{1}} \sum_{j=j_{1}+1}^{l} m^{4} \sum_{y, z \in Z_{m}^{2}}(\tilde{\mathscr{L}}(i-k))^{a}\left(\tilde{\mathscr{L}}\left(j_{1}-i\right)\right)^{b} \\
& \quad \times\left(\tilde{\mathscr{L}}\left(j-j_{1}\right)\right)^{c} p_{k}(y) \bar{p}_{4(i-k)}(z-y) \\
& \quad \times \bar{p}_{4\left(j_{1}-i\right)}(z-y) \bar{p}_{4\left(j-j_{1}\right)}(z-y) \bar{p}_{4(l-j)}(x-z) . \\
& \leqq O(1) n^{-2} \sum_{k=1}^{l} \sum_{j_{1}=k+1}^{l} \sum_{i=k+1}^{j_{1}} \sum_{j=j_{1}+1}^{l}(\tilde{\mathscr{L}}(i-k))^{a} \times\left(\tilde{\mathscr{L}}\left(j_{1}-i\right)\right)^{b}\left(\tilde{\mathscr{L}}\left(j-j_{1}\right)\right)^{c} \\
& \times \tau^{-1}\left(1+m^{2} \frac{\sigma+l-j}{\sigma(l-j+1)}\right)\left(1+m^{2} \frac{k+\sigma+l-j}{k(\sigma+l-j+1)}\right) \\
& \times \frac{l}{k+\sigma+l-j} \bar{p}(4 l, x) \\
& \leqq O(1)\left(n^{-2} l^{2}(\log n / l+1)+n^{-1} m^{2}(\log (l+1))^{2} n^{-2} l^{2} \log (n / l+1)\right. \\
&+\left.n^{-2} m^{4}(\log (l+1))^{3} n^{-1} l \log (n / l+1)\right) \bar{p}(4 l, x) \\
& \leqq O(1)\left(n^{-2} l^{2}\left(\log (n / l+1)+(\log n)^{-1}\right) \bar{p}(4 l, x),\right.
\end{aligned}
$$

if $n^{-1} m^{2} \leqq O(1)(\log n)^{-5 / 2}$. Combining the above estimates we can conclude that

$$
\left|B_{1}\right| \leqq \lambda^{2} \phi(K)\left(n^{-1} l+(\log n)^{-1}+n^{-2} l^{2} \log (n / l+1)\right) \bar{p}(4 l, x) .
$$

To consider $B_{2}$, we set

$$
\begin{aligned}
D_{1}= & \lambda^{2} n^{-2} \sum_{k=1}^{l}\left[\gamma_{k} m^{2} \sum_{y \in Z_{m}^{2}} p_{k}(y) g_{l-k}(x-y)\right. \\
& -\sum_{j_{1}=k+1}^{l} \sum_{i=k+1}^{j_{1}} \sum_{j=j_{1}+1}^{l} m^{4} \sum_{y, z \in Z_{m}^{2}} p_{j}(z) p_{i-k}(z-y) \\
& \left.\times p_{j_{1}-i}(z-y) p_{j-j_{1}}(z-y) g_{l-j}(x-z)\right] \\
D_{2}= & \lambda^{2} n^{-2} \sum_{k=1}^{l} \sum_{j_{1}=k+1}^{l} \sum_{i=k+1}^{j_{1}} \sum_{j=j_{1}+1}^{l} m^{4} \sum_{y, z \in Z_{m}^{2}}\left(p_{j}(z)-p_{k}(y)\right) \\
& \times p_{i-k}(z-y) p_{j_{1}-i}(z-y) p_{j-j_{1}}(z-y) g_{l-j}(x-z) .
\end{aligned}
$$

By the definition of $\gamma_{j}$ we know that

$$
n^{-1} \gamma_{j} \leqq O(1) n^{-1} m^{2}(\log n)^{3}
$$


Thus, by Lemma 3.2 we can show that

$$
\begin{aligned}
\left|D_{1}\right| \leqq & \lambda^{2} n^{-2} \sum_{j=1}^{2} \gamma_{j} m^{2} \sum_{y \in Z_{m}^{2}} p_{j}(y) g_{l-j}(x-y) \\
& +\lambda^{2} n^{-2} \sum_{j=3}^{l} \sum_{k=j}^{n} \sum_{j_{1}=1}^{k-1} \sum_{i=1}^{j_{1}} m^{2} \sum_{y \in Z_{m}^{2}} p_{i}(y) p_{j_{1}-i}(y) p_{k-j_{1}}(y) \\
& \times m^{2} \sum_{z \in Z_{m}^{2}} p_{j}(z) g_{l-j}(x-z) \\
\leqq & \lambda^{2} \phi(K) n^{-2}\left[m^{4}(\log n)^{3}+\sum_{j=3}^{l} \sum_{k=j}^{n} \sum_{j_{1}=1}^{k-1} \sum_{i=1}^{j_{1}}\left(\frac{m^{2}}{i\left(j_{1}-i+1\right)\left(k-j_{1}\right)}\right.\right. \\
& \left.\left.+\frac{1}{i\left(j_{1}-i\right)+i\left(k-j_{1}\right)+\left(j_{1}-i\right)\left(k-j_{1}\right)}\right)\left(m^{2} \frac{l}{j(l-j)}+1\right)\right] \bar{p}(4 l, x) \\
\leqq & \lambda^{2} \phi(K)\left((\log n)^{-1}+n^{-1} l\right) \bar{p}(4 l, x),
\end{aligned}
$$

if $n^{-1} m^{2} \leqq O(1)(\log n)^{-5 / 2}$.

We now consider $D_{2}$. It is easy to show that

$$
\begin{aligned}
\lambda^{2} n^{-2} & \sum_{k=1}^{l} \sum_{j_{1}=k+1}^{l} \sum_{i=k+1}^{j_{1}} \sum_{j=j_{1}+1}^{l} \sum_{y, z \in Z^{2}}\left(p_{j}(z)+p_{k}(y)\right) \\
& \times p_{i-k}(z-y) p_{j_{1}-i}(z-y) p_{j-j_{1}}(z-y) \bar{p}(4(l-j), x-z) \\
\leqq & \lambda^{2} n^{-2} l^{2} \bar{p}(4 l, x) .
\end{aligned}
$$

Then, we can show that the main term in the expression of $D_{2}$ is the following:

$$
\begin{aligned}
& \lambda^{2} n^{-2} \sum_{k=1}^{l} \sum_{j_{1}=k+1}^{l} \sum_{i=k+1}^{j_{1}} \sum_{j=j_{1}+1}^{l} m^{4} \sum_{z \in Z_{m}^{2}}\left(p_{j}(z)-p_{k}(z)\right) \\
& \quad \times p_{i-k}(0) p_{j_{1}-i}(0) p_{j-j_{1}}(0) g_{l-j}(x-z),
\end{aligned}
$$

which is denoted by $D_{2}^{\prime}$. In fact, we can show that

$$
D_{2}^{\prime} \leqq O(1) \lambda^{2} n^{-2} m^{4}(\log n)^{4}+D_{2}^{\prime \prime},
$$

where

$$
\begin{aligned}
D_{2}^{\prime \prime}= & \lambda^{2} n^{-2} \sum_{k=1}^{l} \sum_{j_{1}=k+1}^{l} \sum_{i=k+1}^{j_{1}} \sum_{j=j_{1}+1}^{l} m^{4} \sum_{z \in Z_{m}^{2} \backslash\{0\}}\left(p_{j}(z)-p_{k}(z)\right) \\
& \times p_{i-k}(0) p_{j_{1}-i}(0) p_{j-j_{1}}(0) g_{l-j}(x-z) .
\end{aligned}
$$

Let us first recall an estimate on $p_{n}(x)$. By [20, Proposition 1.2.5] we know that for any given $\alpha \in(1 / 2,2 / 3)$,

$$
\left|p_{n}(x)-\bar{p}_{n}(x)\right| \leqq O(1) n^{3 \alpha-2} \bar{p}_{n}(x),
$$

if $|x| \leqq n^{\alpha}$ and $p_{n}(x)>0$. Using this estimate we can show that

$$
\left|D_{2}^{\prime \prime}\right| \leqq \lambda^{2} \phi(K) \tilde{D}_{2}+\lambda^{2} \phi(K)(\log n)^{-1} \bar{p}(4 l, x),
$$


where

$$
\begin{aligned}
\tilde{D}_{2}= & n^{-2} \sum_{k=1}^{l} \sum_{j=k+2}^{l} \sum_{j_{1}=k+1}^{j-1} \sum_{i=k+1}^{j_{1}} m^{4} \sum_{z \in Z_{m}^{2} \backslash\{0\}}\left|\left(\bar{p}_{j}(z)-\bar{p}_{k}(z)\right)\right| \\
& \times(i-k)^{-1}\left(j_{1}+i+1\right)^{-1}\left(j-j_{1}\right)^{-1} \bar{p}(4(l-j), x-z) .
\end{aligned}
$$

By computation we know that

$$
\begin{aligned}
& n^{-2} m^{2} \sum_{k=1}^{l} \sum_{j_{1}=k+2}^{2 k} \sum_{j_{1}=k+1}^{j-1} \sum_{i=k+1}^{j_{1}} \sum_{z \in Z^{2}} \bar{p}_{2 j}(z) \\
& \quad \times \frac{j-k}{j}(i-k)^{-1}\left(j_{1}-i+1\right)^{-1}\left(j-j_{1}\right)^{-1} \bar{p}(4(l-j), x-z) \\
& \leqq O(1)\left(n^{-2} l^{2}+(\log n)^{-1}\right) \bar{p}(4 l, x)
\end{aligned}
$$

if $n^{-1} m^{2} \leqq O(1)(\log n)^{-5 / 2}$. Moreover, we can show that

$$
\begin{aligned}
& \frac{m^{2}}{n^{2}} \sum_{k=1}^{l / 2} \sum_{j=2 k+1}^{l} \sum_{j_{1}=k+1}^{j-1} \sum_{i=k+1}^{j_{1}} \frac{1}{(i-k)\left(j_{1}-i+1\right)\left(j-j_{1}\right)}\left(1+\frac{l}{l+k-j}\right) \\
& \quad \leqq O(1)\left(n^{-2} l^{2}+(\log n)^{-1}\right)
\end{aligned}
$$

if $n^{-1} m^{2} \leqq O(1)(\log n)^{-5 / 2}$. We remark that

$$
\left|\bar{p}_{j}(z)-\bar{p}_{k}(z)\right| \leqq O(1) \frac{|j-k|}{j} \bar{p}_{2 j}(z)
$$

if $j>k \geqq j / 2$. Then, using (3.1) and (3.2) we can show that

$$
\left|\bar{D}_{2}\right| \leqq \phi(K) \lambda^{2}\left(n^{-2} l^{2}+(\log n)^{-1}\right) \bar{p}(4 l, x) .
$$

Combining the above estimates we can conclude that

$$
\left|D_{2}\right| \leqq \lambda^{2} \phi(K)\left(n^{-2} l^{2}+(\log n)^{-1}\right) \bar{p}(4 l, x) .
$$

The proof of Lemma 3.4 is then complete.

We now consider $A_{l}^{(3)}$. We can show that the first term on the right-hand side of $A_{l}^{(3)}$ is bounded in absolute value by

$$
\begin{aligned}
& \lambda^{3} n^{-3} m^{6} \sum_{k=1}^{l} \sum_{j=k+1}^{l} \sum_{y \in Z_{m}^{2}} E\left[I_{\left\{X_{k}=X_{i}=y\right\}} \exp \left(-\bar{J}_{k+1, j}-\bar{J}_{j+1, l}\right)\right. \\
& \quad \times \sum_{i_{1}=k+1}^{j} \sum_{i_{2}=i_{1}}^{j} \sum_{j_{1}=j+1}^{l} \sum_{j_{2}=j_{1}}^{l}\left(I_{\left\{X_{i_{1}}=X_{j_{1}} \in Z_{m}^{2}, X_{i_{2}}=X_{j_{2}} \in Z_{m}^{2}\right\}}\right. \\
& \left.\left.\quad+I_{\left\{X_{i_{1}}=X_{j_{2}} \in Z_{m}^{2}, X_{i_{2}}=X_{j_{1}} \in Z_{m}^{2}\right\}}\right)\right]
\end{aligned}
$$




$$
\begin{aligned}
\leqq & \lambda^{3} n^{-3} m^{6} \sum_{k=1}^{l} \sum_{j=k+1}^{l} \sum_{y, z_{1}, z_{2} \in Z_{m}^{2}} \sum_{i_{1}=k+1}^{j} \sum_{i_{2}=i_{1}}^{j} \sum_{j_{1}=j+1}^{l} \sum_{j_{2}=j_{1}}^{l}\left(p_{k}(y) g_{i_{1}-k}\left(z_{1}-y\right)\right. \\
& \times g_{i_{2}-i_{1}}\left(z_{2}-z_{1}\right) g_{j-i_{2}}\left(y-z_{2}\right) g_{j_{1}-j}\left(z_{1}-y\right) g_{j_{2}-j_{1}}\left(z_{2}-z_{1}\right) g_{l-j_{2}}\left(z-z_{2}\right) \\
& +p_{k}(y) g_{i_{1}-k}\left(z_{1}-y\right) g_{i_{2}-i_{1}}\left(z_{2}-z_{1}\right) g_{j-i_{2}}\left(y-z_{2}\right) \\
& \left.\times g_{j_{1}-j}\left(z_{2}-y\right) g_{j_{2}-j_{1}}\left(z_{1}-z_{2}\right) g_{l-j_{2}}\left(x-z_{1}\right)\right) .
\end{aligned}
$$

From this we see that the quantity of the form of $n^{-1} m^{2} \sum_{t_{1}=1}^{k_{1}} \sum_{t_{2}=1}^{k_{2}} \sum_{t_{3}=1}^{k_{3}} p_{t_{1}}(0)$ $\times p_{t_{2}}(0) p_{t_{3}}(0)$, which has a bad estimate (see the arguments given in [5, Sect. 3]), does not appear on the right-hand side of the above estimate. Thus, using the arguments given before we can show that the first term on the right-hand side of $A_{l}^{(3)}$ can be bounded in absolute value by:

$$
\lambda^{3} \phi(K)\left(n^{-2} l^{2}+(\log n)^{-1}\right) \bar{p}(4 l, x) .
$$

By the same reason as before we can show that the second term on the right-hand side of $A_{l}^{(3)}$ is bounded in absolute value by:

$$
\lambda^{4} \phi(K)\left(n^{-2} l^{2}+(\log n)^{-1}\right) \bar{p}(4 l, x),
$$

which proves that

$$
\left|A_{l}^{(3)}\right| \leqq\left(\lambda^{3}+\lambda^{4}\right) \phi(K)\left(n^{-2} l^{2}+(\log n)^{-1}\right) \bar{p}(4 l, x) .
$$

We now consider $\mathscr{A}_{l}^{(2)}$. Let

$$
\begin{aligned}
& \xi_{1}=\lambda n^{-1} \beta_{k} m^{2} I_{\left\{X_{k} \in Z_{m}^{2}\right\}}-\lambda^{2} n^{-2} \gamma_{k} m^{2} I_{\left\{X_{k} \in Z_{m}^{2}\right\}}, \\
& \xi_{2}=\lambda n^{-1} \sum_{j=k+1}^{l} m^{2} \sum_{y \in Z_{m}^{2}} I_{\left\{X_{k}=X_{j}=y\right\}} .
\end{aligned}
$$

It is clear that

$$
\left|\xi_{1}\right| \leqq O(1)(\log n)^{-3 / 2},
$$

if $n^{-1} m^{2} \leqq O(1)(\log n)^{-5 / 2}$, and

$$
\begin{aligned}
\mathscr{A}_{l}^{(2)} & =\sum_{k=1}^{l} E \exp \left(-\bar{J}_{k+1, l}\right)\left(1-\exp \left(-\xi_{2}+\xi_{1}\right)-\left(\xi_{2}-\xi_{1}\right)\right) \delta\left(X_{l}, x\right) \\
& \geqq \sum_{k=1}^{l} E \exp \left(-\bar{J}_{k+1, l}\right)\left(1-\exp \left(\xi_{1}\right)\left(1-\xi_{2}\right)-\left(\xi_{2}-\xi_{1}\right)\right) \delta\left(X_{l}, x\right) \\
& \geqq \sum_{k=1}^{l} E \exp \left(-\bar{J}_{k+1, l}\right)\left(1-\left(1+\xi_{1}+O\left(\xi_{1}^{2}\right)\right)\left(1-\xi_{2}\right)-\left(\xi_{2}-\xi_{1}\right)\right) \delta\left(X_{l}, x\right) \\
& =\sum_{k=1}^{l} E \exp \left(-\bar{J}_{k+1, l}\right)\left(\xi_{1} \xi_{2}+O(1) \xi_{1}^{2}+O(1) \xi_{1}^{2} \xi_{2}\right) \delta\left(X_{l}, x\right) .
\end{aligned}
$$

Similarly, we can show that

$$
\mathscr{A}_{l}^{(2)} \leqq \sum_{k=1}^{l} E \exp \left(-\bar{J}_{k+1, l}\right)\left(\xi_{1} \xi_{2}+O(1)\left(\xi_{1}^{2}+\xi_{2}^{2}\right)+O(1) \xi_{1}^{2} \xi_{2}\right) \delta\left(X_{l}, x\right)
$$


By computation we can easily show that

$$
\begin{aligned}
& \sum_{k=1}^{l} E \exp \left(-\bar{J}_{k+1, l}\right) \xi_{1}^{2} \delta\left(X_{l}, x\right) \leqq \phi(K)\left(\frac{l}{n}(\log n)^{-1 / 2}+(\log n)^{-2}\right) \bar{p}(4 l, x) \\
& \sum_{k=1}^{l} E \exp \left(-\bar{J}_{k+1, l}\right) \xi_{2} \delta\left(X_{l}, x\right) \leqq \lambda \phi(K)\left((\log n)^{-1 / 2}+\frac{l}{n} \log (n / l+1)\right) \bar{p}(4 l, x)
\end{aligned}
$$

and

$$
\sum_{k=1}^{l} E \exp \left(-\bar{J}_{k+1, l}\right) \xi_{2}^{2} \delta\left(X_{l}, x\right) \leqq \lambda^{2} \phi(K)\left((\log n)^{-1}+n^{-2} l^{2}\right) \bar{p}(4 l, x)
$$

Using the above estimates we can show that

$$
\left|\mathscr{A}_{l}^{(2)}\right| \leqq \lambda^{2} \phi(K)\left((\log n)^{-1}+n^{-2} l^{2}\right) \bar{p}(4 l, x) .
$$

Proof of Proposition 3.1. Having the preparations given before, by a similar argument as in the proof of [2, Theorem 3.1] we can easily prove Proposition 3.1.

In fact, we need only to show that

$$
\begin{aligned}
& \mid n^{-1} \sum_{l=1}^{i} \sum_{k=1}^{l} \sum_{j=k+1}^{l} m^{2} \sum_{y \in Z_{m}^{2}} p_{k}(y) g_{l-j}(y) p_{j-k}(0) \\
& \quad-n^{-1} \sum_{l=1}^{i} \sum_{k=1}^{l} \sum_{j=1}^{n} p_{j}(0) m^{2} \sum_{y \in Z_{m}^{2}} p_{k}(y) g_{l-k}(y) \mid \\
& \leqq \phi(K)\left(n^{-1} m^{2}(\log (i+1))^{2} \log (n / i+1)+1\right) .
\end{aligned}
$$

Indeed, we can show that the left-hand side of (3.3) is less than

$$
\begin{aligned}
& n^{-1} m^{2} \phi(K) \sum_{k=1}^{i} \sum_{l=1}^{i-k} \sum_{y \in Z_{m}^{2}} p_{k}(y) g_{l}(y) \sum_{j=i-k-l}^{n} p_{j}(0) \\
& \quad \leqq n^{-1} \phi(K)\left[\sum_{k=1}^{i} \sum_{l=1}^{i-k} \frac{m^{2}}{k l} \log \frac{n}{i-k-l+1}+\sum_{k=1}^{i} \sum_{l=1}^{i-k} \frac{1}{k+l} \log \frac{n}{i-k-l+1}\right] \\
& \quad \leqq \phi(K)\left(n^{-1} m^{2}(\log (i+1))^{2} \log (n / i+1)+1\right)
\end{aligned}
$$

which proves (3.3). Since $n^{-1} m^{2} \leqq O(1)(\log n)^{-5 / 2}$, we know by (3.3) that

$$
K(m, n) \leqq \lambda \phi(K(m, n)),
$$

and so there are constants $c_{4} \in(0, \infty)$ and $\lambda_{0}>0$ such that

$$
K(m, n) \leqq c_{4} \lambda, \quad \forall \lambda \in\left[0, \lambda_{0}\right] .
$$

In other words, we have

$$
\left|g_{k}(x)-p(k, x)\right| \leqq O(1) \lambda \bar{p}(4 k, x), \quad k \in[1, n], \forall x \in Z^{2}, \lambda \in\left[0, \lambda_{0}\right] .
$$

This implies the desired result by choosing a sufficient small $\lambda_{0}>0$. 
Remark 3.5. From the proof of Proposition 3.1 given above one can see that the constant $\lambda_{0} \in(0, \infty)$ depends on the factor $\lim \sup _{n \rightarrow \infty} n^{-1} m^{2}(\log n)^{5 / 2}$. However, one can also see that the constant $\lambda_{0}$ given in Proposition 3.1 is independent of $m$ and $n$, if $\lim \sup _{n \rightarrow \infty} n^{-1} m^{2}(\log n)^{5 / 2}=0$.

\section{On the Large Coupling Constants}

In this section we always assume $\lim _{n \rightarrow \infty} n^{-1} m^{2}(\log n)^{5 / 2}=0$. The main aim of this section is to prove that the assertion given in Proposition 3.1 also holds for all finite and positive coupling constants, i.e.

Proposition 4.1. For any given $\lambda \in(0, \infty)$ there are constants $c_{1}, c_{2} \in(0, \infty)$ such that

$$
c_{1} \leqq E \exp \left(-\bar{S}_{m_{n}, n}(\lambda)\right) \leqq c_{2} .
$$

Remark that

$$
\bar{J}_{k, l}^{m, 2 \lambda}=\bar{S}_{m, n}(\lambda)
$$

Let

$$
G(A)=\int_{A} \exp \left(-\bar{J}_{1, n}^{m, 2 \lambda}\right) d P, \quad \forall A \subset Z^{2} .
$$

Let $E_{x}$ be the expectation with respect to $P_{x}$. The next lemma plays a key role in the proof of Proposition 4.1.

Lemma 4.2. There are constants $c_{3}, c_{4} \in(0, \infty)$ and $\lambda_{0}>0$ such that

$$
\begin{gathered}
E_{x} \exp \left(-\bar{J}_{1, n}^{m, 2 \lambda}\right) \leqq c_{3}, \quad \lambda \in\left[0, \lambda_{0}\right], \\
E_{x} I_{\left\{X_{l}=y\right\}} \exp \left(-\bar{J}_{1, l}^{m, 2 \lambda}\right) \leqq O(1) \bar{p}\left(c_{4} l, y-x\right), \quad \forall \lambda \in\left[0, \lambda_{0}\right], \quad l \geqq n / 2
\end{gathered}
$$

for all $x, y \in Z^{2}$.

Proof. It was proved in the proof of Proposition 3.1 that (4.1) and (4.2) are correct for $x=0$. Without loss of generality, we may assume $x \in S_{n}(0) \backslash\{0\}$. Let

$$
\tau=\inf \left\{k \geqq 0: X_{k} \in Z_{m}^{2}\right\} .
$$

By the strong Markov property we can show that

$$
\begin{aligned}
& E_{x} I_{\left\{X_{l}=y\right\}} \exp \left(-\bar{J}_{1, l}\right) \\
& =E_{x} I_{\left\{\tau>l, X_{l}=y\right\}}+E_{x} I_{\left\{\tau=l, X_{l}=y\right\}} \exp \left(-\lambda \beta n^{-1} m^{2}+\lambda^{2} n^{-2} m^{2} \gamma\right) \\
& \quad+\sum_{z \in Z_{m}^{2}} E_{x} I_{\left\{\tau<l, X_{\tau}=z\right\}} E_{z}\left(\exp \left(-\bar{J}_{1, l-\tau}\right) I_{\left\{X_{l-\tau}=y\right\}}\right) .
\end{aligned}
$$

By Proposition 3.1 we know that there is a constant $\lambda_{0}>0$ such that

$$
\begin{gathered}
E_{z} \exp \left(-\bar{J}_{1, l-\tau}\right) I_{\left.X_{\{l-\tau}=y\right\}} \leqq O(1) \bar{P}(4(l-\tau), y-z), \\
E_{z} \exp \left(-\bar{J}_{1, l-\tau}\right) \geqq O(1),
\end{gathered}
$$


if $\lambda \in\left[0, \lambda_{0}\right]$ and $z \in Z_{m}^{2}$. Using the last estimate and (4.3) we can easily show that (4.2) is true.

We now prove (4.1). Without loss of generality, we may prove (4.1) only for $l=n$. We first derive an estimate on the stopping time $\tau$. For any $x \in Z^{2}$ we have

$$
P_{x}(\tau>T)=P_{x}\left(\bigcap_{i=1}^{T}\left\{X_{i} \notin Z_{m_{n}}^{2}\right\}\right) .
$$

Let $l_{n}=\inf \left\{k \geqq 1: k m_{n}^{2} \leqq T<(k+1) m_{n}^{2}\right\}$. Then

$$
\begin{aligned}
& P_{x}(\tau>T) \leqq P_{x}\left(\bigcap_{k=1}^{l_{n}} \bigcap_{i=(k-1) m_{n}^{2}+1}^{k m_{n}^{2}}\left\{X_{i} \notin Z_{m_{n}}^{2}\right\}\right) \\
& \quad=E_{x}\left[\bigcap_{k=1}^{l_{1}-1} \bigcap_{i=(k-1) m_{n}^{2}+1}^{k m_{n}^{2}}\left\{X_{i} \notin Z_{m_{n}}^{2}\right\}, P_{X_{\left(l_{n}-1\right) m_{n}^{2}+1}}\left(\tau>m_{n}^{2}\right)\right] .
\end{aligned}
$$

By [21, Theorem 3.4] we can show that if $y \in S_{n}(0)$,

$$
P_{y}\left(\tau \leqq m_{n}^{2}\right) \geqq c_{5}\left(\log m_{n}\right)^{-1}
$$

for some constant $c_{5} \in(0, \infty)$. By the symmetry property of $\left\{X_{n}\right\}$ we know that

$$
\begin{aligned}
P_{X_{\left(l_{n}-1\right) m_{n}^{2}+1}}\left(\tau>m_{n}^{2}\right) & =P_{X_{\left(l_{n}-1\right) m_{n}^{2}+1}-\eta\left(X_{\left(l_{n}-1\right) m_{n}^{2}+1}\right)}\left(\tau>m_{n}^{2}\right) \\
& \leqq 1-c_{5}\left(\log m_{n}\right)^{-1} .
\end{aligned}
$$

By (4.4) we can show that

$$
P_{x}(\tau>T) \leqq\left(1-c_{5}\left(\log m_{n}\right)^{-1}\right)^{l_{n}} .
$$

By (4.5) we know that for any given $M \geqq 1$ there is a constant $M_{1} \in(0, \infty)$ such that

$$
P_{x}\left(\tau>T_{n}\right) \leqq \exp (-M \log n)
$$

if $T_{n} \in\left[M_{1} m_{n}^{2}(\log n)^{2},\left(M_{1}+1\right) m_{n}^{2}(\log n)^{2}\right]$.

On the other hand, we can show that

$$
E_{0} \sum_{k=1}^{l} I_{\left\{X_{k} \in Z_{m}^{2}\right\}} \leqq O(1)\left(l m^{-2} \vee 1\right) .
$$

Hence, there is a constant $c_{6} \in(0, \infty)$ such that

$$
E\left(\sum_{k=1}^{l} I_{\left\{X_{k} \in Z_{m}^{2}\right\}}\right)^{p} \leqq p ! c_{6}^{p}\left(l m^{-2} \vee 1\right)^{p}, \quad \forall p \geqq 0 .
$$

From this we can see that there is a constant $c_{7} \in(0, \infty)$ such that

$$
E_{0} \exp \left(\lambda n^{-1} \sum_{k=1}^{n-\tau} \beta_{k} m^{2} I_{\left\{X_{k} \in Z_{m}^{2}\right\}}\right) \leqq \exp \left(c_{7} \log n\right), \quad \lambda \in\left[0, \lambda_{0}\right] .
$$

We now prove that the following holds under the assumption $|x-y|^{2} \leqq$ $O(1) n(\log n)^{1 / 2}$ :

$$
E_{x} I_{\left\{X_{n}=y\right\}} \exp \left(-\bar{J}_{1, n}^{m, 2 \lambda}\right) \leqq O(1) \bar{p}\left(c_{4} n, y-x\right)
$$


Indeed, if $|x-y|^{2} \leqq O(1) n$, by (4.3) and (4.5) we can easily show that the lefthand side of (4.7) is less than

$$
\begin{aligned}
E_{x} I_{\left\{\tau>n / 2, X_{n}=y\right\}} \exp \left(-\bar{J}_{1, n}^{m, 2 \lambda}\right)+E_{x} I_{\{\tau \leqq n / 2\}} E_{X_{\tau}} \exp \left(-\bar{J}_{1, n-\tau}^{m, 2 \lambda}\right) I_{\left\{X_{n-\tau}=y\right\}} \\
\quad \leqq P_{x}^{1 / 2}(\tau>n / 2) E^{1 / 2} \exp \left(-2 \bar{J}_{1, n}^{m, 2 \lambda}\right)+O(1) E_{x} I_{\{\tau \leqq n / 2\}} \bar{p}\left(4(n-\tau), y-X_{\tau}\right) \\
\quad \leqq O(1) \exp \left(-c_{8}(\log n)^{3 / 2}\right) \exp \left(c_{7} \log n\right)+O(1) n^{-1} \\
\quad \leqq O(1) \bar{p}(n, y-x), \quad \lambda \in\left[0, \lambda_{0}\right]
\end{aligned}
$$

for some constant $c_{8} \in(0, \infty)$. Hence (4.7) is true if $|x-y|^{2} \leqq O(1) n$. We now assume $|x-y|^{2} \geqq K n$ for some large $K \geqq 1$. In this case, we know that the lefthand side of (4.7) is less than

$$
\begin{aligned}
& O(1) \exp \left(-c_{8}(\log n)^{3 / 2}\right)+O(1) \bar{p}(64 n, x-y) \\
& \quad+E_{x} I_{\{\tau \leqq n / 2\}} I_{\left\{\left|X_{\tau-y}\right| \leqq|x-y| / 8\right\}} E_{X_{\tau}} \exp \left(-\bar{J}_{1, n-\tau}^{m, 2 \lambda}\right) I_{\left\{X_{n-\tau}=y\right\}} .
\end{aligned}
$$

We need only to consider the last term on the right-hand side of the above estimate, which is denoted by $I$. Indeed, we have

$$
\begin{aligned}
I \leqq & O(1) P_{x}^{1 / 2}\left(\tau>T_{n}\right)+E_{x}\left[I_{\{\tau \leqq} T_{n} I_{\left\{\left|X_{\tau-y}\right| \leqq|x-y| / 8\right\}}\right. \\
& \left.\times E_{X_{\tau}} \exp \left(-\bar{J}_{1, n-\tau}^{m, 2 \lambda}\right) I_{\left\{X_{n-\tau}=y\right\}}\right] \\
\leqq & O(1) n^{-M / 2}+O(1) n^{-1} P_{0}\left(\max _{1 \leqq i \leqq\left(M_{1}+1\right) m_{n}^{2}(\log n)^{2}}\left|X_{i}\right| \geqq 7|x-y| / 8\right) \\
\leqq & O(1) n^{-M / 2}+O(1) n^{-1} \exp \left(-c_{9} \frac{|x-y|}{m_{n}^{2}(\log n)^{2}}\right) \\
\leqq & O(1) \bar{p}\left(c_{10} n, x-y\right),
\end{aligned}
$$

for some constants $c_{9}, c_{10} \in(0, \infty)$, where $\frac{n}{m_{n}^{2}(\log n)^{2}} \geqq(\log n)^{1 / 2}$ and $|x-y|^{2} \leqq$ $O(1) n(\log n)^{1 / 2}$.

We now prove that (4.7) holds under the assumption: $K n(\log n)^{1 / 2} \leqq|x-y|^{2} \leqq$ $O(1) n(\log n)^{3 / 2}$. Indeed, by an argument similar to the one given before we can show that

$$
\begin{aligned}
I \leqq & O(1) P_{x}^{1 / 2}(\tau>n / 2)+E_{x}\left[I_{\{\tau \leqq n / 2\}} I_{\left\{\left|X_{\tau-y}\right| \leqq|x-y| / 8\right\}}\right. \\
& \left.\times E_{X_{\tau}} \exp \left(-\bar{J}_{1, n-\tau}^{m, 2 \lambda}\right) I_{\left\{X_{n-\tau}=y\right\}}\right] \\
\leqq & O(1) \exp \left(-c_{8}(\log n)^{3 / 2}\right)+O(1) n^{-1} \exp \left(-c_{11} \frac{|x-y|^{2}}{n}\right) \\
\leqq & O(1) \bar{p}\left(c_{12} n, x-y\right)
\end{aligned}
$$

for some constant $c_{11}, c_{12} \in(0, \infty)$. Hence, (4.7) is also true in this case. We now prove that (4.7) holds under the assumption: $|x-y|^{2} \geqq K n(\log n)^{3 / 2}$ for some large $K \geqq 1$. Indeed, by (4.6) and the Hölder inequality we have

$$
I \leqq O(1) P_{x}^{1 / 2}\left(X_{n}=y\right) \exp \left(c_{7} \log n\right) \leqq O(1) \bar{p}\left(c_{13} n, x-y\right)
$$

for some constant $c_{13} \in(0, \infty)$.

Concluding the above estimates we obtain the desired result. 
We now use Lemma 4.2 to prove the next two lemmas which will be used in the proof of Proposition 4.1.

Lemma 4.3. Assume that $\lim _{n \rightarrow \infty} m_{n}^{2} n^{-1}(\log n)^{5 / 2}=0$.

(i) For any given $\lambda \in[0, \infty)$ there are constants $c_{14}, c_{15} \in(0, \infty)$ such that

$$
G\left(X_{l}=x\right) \leqq c_{14} \bar{p}\left(c_{15} l, x\right), \quad \forall x \in Z^{2}, l \leqq n
$$

(ii) For any given $\lambda \in[0, \infty)$ there is a constant $c_{16} \in(0, \infty)$ which is independent of $m$ and $n$ such that

$$
E \exp \left(-\bar{J}_{1, n}^{m_{n}, \lambda}\right) \leqq c_{16}
$$

Proof. (i) By Proposition 3.1 we know that there is a constant $\lambda_{0}>0$ such that

$$
g_{l}(x) \leqq O(1) \bar{p}(4 l, x), \quad \forall x \in Z^{2}, l \geqq 1, \lambda \in\left[0, \lambda_{0}\right]
$$

By definition we can easily show that

$$
\bar{J}_{1, n} \geqq \bar{J}_{1, l}+\bar{J}_{l+1, n}, \quad l \in[1, n] .
$$

Thus, by Lemma 4.2 we can show that

$$
\begin{aligned}
G\left(X_{l}=x\right) & \leqq \int_{\Omega} \exp \left(-\bar{J}_{1, l}\right) I_{\left\{X_{l}=x\right\}} \exp \left(-\bar{J}_{l+1, n}\right) d P \\
& \leqq \int_{\Omega} \exp \left(-\bar{J}_{1, l}\right) I_{\left\{X_{l}=x\right\}} E_{x} \exp \left(-\bar{J}_{1, n-l}\right) \\
& \leqq O(1) g_{l}(x) \leqq O(1) \bar{p}(4 l, x), \quad \forall x \in Z^{2}, \lambda \in\left[0, \lambda_{0}\right] .
\end{aligned}
$$

We now prove that (4.8) holds for $\lambda \in\left[0,2 \lambda_{0}\right]$. As in the proof of [2, Lemma 4.1] we may prove (4.8) only for $l=n$. Without loss of generality, we may assume $n=2^{n_{1}}$ and $m=2^{m_{1}}$. Then we have (see the proof of [2, Lemma 4.1])

$$
\begin{aligned}
G\left(X_{n}=x\right) & =g_{2^{n_{1}}}^{2^{m_{1}}, 2 \lambda}(x) \leqq O(1) \sum_{y \in Z^{2}} g_{2^{n_{1}}-1}^{2^{m_{1}}, \lambda}(y) g_{2^{n_{1}-1}}^{2^{m_{1}}, \lambda}(x-y) \\
& \leqq O(1) \bar{p}\left(c_{17} 2^{n_{1}}, x\right)
\end{aligned}
$$

for some constant $c_{17} \in(0, \infty)$, which implies the desired result.

(ii) This is an immediate consequence of (i).

For convenience, we assume $n=2^{n_{1}}$. Let

$$
\mathscr{N}_{i, n_{1}}=\#\left\{k \leqq 2^{n_{1}}: 2^{(i-1) / 2}-1 \leqq\left|X_{k}-X_{2^{n_{1}-1}}\right| \leqq 2^{i / 2}, X_{k} \in Z_{m}^{2} \backslash\{0\}\right\} .
$$

Lemma 4.4. There is a constant $c_{18} \in(0, \infty)$ such that

$$
G\left(\bigcup_{i=1}^{\infty}\left\{\mathscr{N}_{i, n_{1}} \geqq M\left(\left(n-i_{1}\right) \vee 1\right)^{3} 2^{i} m^{-2} \exp \left(-2^{i-n_{1}-2}\right)\right) \leqq C_{18} M^{-1}\right.
$$

Proof. By Lemma 4.3 we know that

$$
G\left(X_{k}=y\right) \leqq O(1) \bar{p}\left(c_{15} k, y\right),
$$


and so

$$
\sum_{k=1}^{2^{n_{1}-1}} G\left(X_{k}=y\right) \leqq O(1) \log \left(\frac{2^{n_{1}-1}}{|x|^{2}} \vee 2\right) \exp \left(-\frac{|x|^{2}}{2^{n_{1}}}\right) .
$$

Then, as in the proof of Lemma 4.3 we can show that

$$
\begin{aligned}
E_{G} \mathscr{N}_{i, n_{1}} & \leqq \sum_{k=1}^{2^{n_{1}-1}} \sum_{\substack{2^{(i-1) / 2} \\
y \in Z_{m}^{2} \backslash\{0\}}} G\left(X_{k}=y, X_{2^{n} 1^{-1}}=x\right) \\
& \leqq \sum_{k=1}^{2^{n_{1}-1}} \sum_{\substack{2^{(i-1) / 2} \\
y \in Z_{m}^{2} \backslash\{0\}}} G\left(X_{k}=y\right) G\left(X_{2^{n}-1}-k=x-y\right) .
\end{aligned}
$$

By Lemma 4.3 we have

$$
\begin{aligned}
& E_{G} \mathscr{N}_{i, n_{1}} \leqq O(1) \sum_{k=1}^{2^{n_{1}-1}} \sum_{\substack{2^{(i-1) / 2} \\
y \in Z_{m}^{2} \backslash\{0\}}} \bar{p}\left(c_{15} k, y\right) \bar{p}\left(c_{15}\left(2^{n_{1}-1} k, x-y\right)\right. \\
& \leqq O(1) \sum_{k=1}^{2^{n_{1}-1}} \sum_{\substack{2^{(i-1) / 2}\left(-1 \leqq|z| \leqq 2^{i / 2} \\
y \in Z_{m}^{2} \backslash\{0\}\right.}} \bar{p}\left(c_{15} k, y\right) \bar{p}\left(c_{15}\left(2^{n_{1}-1}-k\right), z\right) \\
& \leqq O(1) m^{-2} \sum_{k=1}^{2^{n_{1}-1}} \sum_{2^{(i-1) / 2}} \bar{p} \bar{p}\left(c_{15}\left(2^{n_{1}-1}-k\right), z\right) \\
& \leqq O(1) m^{-2}\left(\left(n-i_{1}\right) \vee 1\right) 2^{i} \exp \left(-2^{i-n_{1}-1}\right),
\end{aligned}
$$

which implies the desired result.

To state the next lemma, we let $\left\{\tilde{X}_{n}\right\}_{n \geqq 0}$ be a simple random walk in $Z^{2}$ independent of $\left\{X_{n}\right\}_{n \geqq 0}$. Let $\left\{\tilde{P}_{x}\right\}$ be the probability law of $\left\{\tilde{X}_{n}\right\}$. Let

where

$$
\tilde{G}_{x}(A)=\int_{A} \exp \left(-\tilde{J}_{1,2^{n_{1}-1}}^{m, 2 \lambda}(x)\right) d \tilde{P}_{0}
$$

$$
\begin{aligned}
\tilde{J}_{1,2^{n_{1}}-1}^{m, 2 \lambda}(x)= & 2 \lambda m^{2} 2^{-\left(n_{1}-1\right)} \sum_{i=1}^{2^{n_{1}-1}} \sum_{j=i+1}^{2^{n_{1}-1}} I_{\left\{\tilde{X}_{i}=\tilde{X}_{j} \in Z_{m}^{2}-x\right\}} \\
& -\left(2 \lambda m^{2} 2^{-\left(n_{1}-1\right)} \beta-4 \lambda^{2} m^{2} 2^{-2\left(n_{1}-1\right)} \gamma\right) \sum_{i=1}^{2^{n_{1}-1}} I_{\left\{\tilde{X}_{i} \in Z_{m}^{2}-x\right\}},
\end{aligned}
$$

and $Z_{m}^{2}-x=\left\{z-x: z \in Z_{m}^{2}\right\}$. By Lemma 4.3 we know that

$$
\tilde{G}_{x}\left(\tilde{X}_{l}=y\right)=E_{x} I_{\left\{X_{l}=y-x\right\}} \exp \left(-\bar{J}_{1,2^{n_{1}-1}}^{m, 2 \lambda}\right) \leqq O(1) \bar{p}\left(c_{15} l, y\right) .
$$

Lemma 4.5. Let $\left\{x(1), \ldots, x\left(2^{n_{1}-1}\right)\right\}$ be a given set. Suppose that there is a constant $M \geqq 1$ such that

$$
\begin{aligned}
& \sum_{k=1}^{2^{n_{1}-1}} I_{\left\{x(k): 2^{(i-1) / 2}-1 \leqq\left|x(k)-x\left(2^{n_{1}-1}\right)\right| \leqq 2^{i / 2}, x(k) \in Z_{m}^{2} \backslash\{0\}\right\}} \\
& \quad \leqq M m^{-2}\left(\left(n_{1}-i\right) \vee 1\right)^{3} 2^{i} \exp \left(-2^{i-n_{1}-2}\right), \quad i=1,2, \ldots .
\end{aligned}
$$


Then, there is a constant $c_{19} \in(0, \infty)$ such that

$$
\begin{aligned}
& \tilde{G}_{x\left(2^{n_{1}-1}\right)}\left(\sum_{j=1}^{2^{n_{1}-1}} \sum_{k=1}^{2^{n_{1}-1}} I_{\left\{\tilde{X}_{j}=x(k)-x\left(2^{n_{1}-1}\right), x(k) \in Z_{m}^{2} \backslash\{0\}\right\}} \geqq K m^{-2} 2^{n_{1}-1}\right) \\
& \quad \leqq c_{19} K^{-1} M, \quad \forall k \geqq 1 .
\end{aligned}
$$

Proof. As in the proof of Lemma 4.4, by (4.9) we can show that

$$
\begin{aligned}
& E_{\tilde{G}_{x\left(2^{n_{1}}-1\right.}} \sum_{j=1}^{2^{n_{1}-1}} \sum_{k=1}^{2^{n_{1}-1}} I_{\left\{\tilde{X}_{j}=x(k)-x\left(2^{n_{1}-1}\right), x(k) \in Z_{m}^{2} \backslash\{0\}\right\}} \\
& \leqq \sum_{i=1}^{\infty} \sum_{k=1}^{2^{n_{1}-1}} I_{\left\{2^{(i-1) / 2}-1 \leqq\left|x(k)-x\left(2^{n^{n}-1}\right)\right| \leqq 2^{i / 2}, x(k) \in Z_{m}^{2} \backslash\{0\}\right\}} \\
& \times \sum_{j=1}^{2^{n_{1}-1}} \tilde{G}_{x\left(2^{n_{1}-1}\right)}\left(\tilde{X}_{j}=x(k)-x\left(2^{n_{1}-1}\right)\right) \\
& \leqq O(1) \sum_{i=1}^{\infty} \sum_{k=1}^{2^{n_{1}-1}} I_{\left\{2^{(i-1) / 2}-1 \leqq\left|x(k)-x\left(2^{n_{1}-1}\right)\right| \leqq 2^{i / 2}, x(k) \in Z_{m}^{2} \backslash\{0\}\right\}} \\
& \quad \times \log \left(\frac{2^{n_{1}-1}}{2^{i-1}} \vee 2\right) \exp \left(-2^{i-n_{1}-1}\right) \\
& \leqq O(1) M m^{-2} 2^{n_{1}-1} .
\end{aligned}
$$

From this one easily obtains the desired result.

We are now in a position to complete the proof of Proposition 4.1. Proof of Proposition 4.1. It is clear that

$$
\begin{aligned}
G(\Omega)= & \int_{\Omega} \exp \left(-\bar{J}_{1,2^{n_{1}-1}}^{m, 2 \lambda}\right) \exp \left(-\bar{J}_{2^{n_{1}-1}+1,2^{n_{1}}}^{m}\right) \\
& \times \exp \left(-2 \lambda m^{2} 2^{-n_{1}} \sum_{i=1}^{2 n_{1}-1} \sum_{j=2^{n_{1}-1}+1}^{2^{n_{1}}} I_{\left\{X_{i}=X_{j} \in Z_{m}^{2}\right\}}\right) d P \\
= & \int_{\Omega} \exp \left(-\bar{J}_{1,22^{n_{1}-1}}^{m, 2 \lambda}\right) d \tilde{G}_{X\left(2^{n_{1}-1}\right)} d P \\
& \times \exp \left(-2 \lambda m^{2} 2^{-n_{1}} \sum_{i=1}^{2^{n_{1}-1}} \sum_{j=1}^{2^{n_{1}-1}} I_{\left\{X_{i}-X_{2^{n_{1}-1}-1}=\tilde{X}_{j} \in Z_{m}^{2}-X_{2^{n_{1}}-1}\right\}}\right) \\
= & \int_{\Omega} \exp \left(-2 \lambda m^{2} 2^{-n_{1}} \sum_{i=1}^{2^{n_{1}-1}} \sum_{j=1}^{2^{n-1-1}} I_{\left\{-X_{2^{n_{1}-1}}=\tilde{X}_{j}, X_{i}=0\right\}}\right) d \tilde{G}_{X\left(2^{n_{1}-1}\right)} d G^{\prime} \\
& \times \exp \left(-2 \lambda m^{2} 2^{-n_{1}} \sum_{i=1}^{2^{n_{1}-1}} \sum_{j=1}^{2^{n_{1}-1}} I_{\left\{X_{i}-X_{2^{n_{1}-1}-1} \tilde{X}_{j}, X_{i} \in Z_{m}^{2} \backslash\{0\}\right\}}\right)
\end{aligned}
$$


where

$$
G^{\prime}(A)=\int_{A} \exp \left(-\bar{J}_{1,2^{n_{1}}-1}^{m, 2 \lambda}\right) d P
$$

Let

$$
\begin{aligned}
& A(M)=\bigcap_{i=1}^{\infty}\left\{\mathscr{N}_{i, n_{1}}<M\left(\left(n_{1}-i\right) \vee 1\right)^{3} 2^{i} m^{-2} \exp \left(-2^{i-n_{1}-2}\right)\right\}, \\
& \tilde{A}(K)=\left\{\sum_{j=1}^{2^{n_{1}-1}} \sum_{i=1}^{2^{n_{1}-1}} I_{\left\{X_{i}-X_{2^{n_{1}-1}}=\tilde{X}_{j}, X_{i} \in Z_{m}^{2} \backslash\{0\}\right\}}<K m^{-2} 2^{n_{1}-1}\right\}, \\
& B(M)=\left\{\sum_{i=1}^{2^{n_{1}-1}} I_{\left\{X_{i}=0\right\}}<M n_{1}\right\} \text {, } \\
& \tilde{B}(M)=\left\{\sum_{j=1}^{2^{n_{1}-1}} I_{\left\{-X_{2^{n_{1}-1}}=\tilde{X}_{j}\right\}}<M n_{1}\right\} .
\end{aligned}
$$

Then, by Lemma 4.3 we can show that

$$
G^{\prime}\left(B^{c}(M)\right) \leqq M^{-1} n_{1}^{-1} \sum_{i=1}^{2^{n_{1}-1}} G\left(X_{i}=0\right) \leqq O(1) M^{-1}
$$

Similarly, by (4.9) we can show that

$$
\tilde{G}_{X\left(2^{n_{1}-1}\right)}\left(\tilde{B}^{c}(M)\right) \leqq O(1) M^{-1} .
$$

Recalling the assumption: $m^{2} 2^{-n_{1}} \leqq O(1) n_{1}^{5 / 2}$, by Lemma 4.4 and Lemma 4.5 we can show that there is a constant $c_{20} \in(0, \infty)$ such that

$$
\begin{aligned}
G(\Omega) \geqq & \int_{A(M) \cap \tilde{A}(K) \cap B(M) \cap \tilde{B}(M)} d \tilde{G}_{X\left(2^{n_{1}-1}\right)} d G^{\prime} \\
& \times \exp \left(-2 \lambda m^{2} 2^{-n_{1}} M^{2} n_{1}\right) \exp (-2 \lambda K) \\
\geqq & c_{20} \int_{A(M) \cap B(M)} \tilde{G}_{X\left(2^{n_{1}-1}\right)}(\tilde{A}(K) \cap \tilde{B}(M)) d P \\
\geqq & c_{20} \int_{A(M) \cap B(M)}\left(1-O(1) M^{-1}-O(1) K^{-1} M\right) d P \\
\geqq & c_{20}\left(1-O(1) M^{-1}-O(1) K^{-1} M\right)\left(1-O(1) M^{-1}\right) .
\end{aligned}
$$

Hence, if $M \geqq 1$ and $K \geqq M$ are chosen to be large enough, one obtains

$$
G(\Omega) \geqq c_{21}
$$

for some constant $c_{21} \in(0, \infty)$ which is independent of $m$ and $n=2^{n_{1}}$. This completes the proof of Proposition 4.1.

\section{Proof of Theorem 2.1}

By Proposition 4.1 and (4.2) we can easily show that $\left\{v_{m_{n}, n, \lambda}\right\}_{n \geqq 1}$ is tight for each $\lambda \in[0, \infty)$, provided $\lim \sup _{n \rightarrow \infty} n^{-1} m_{n}^{2}(\log n)^{5 / 2}=0$. In fact, by Proposition 3.1 we 
also know that there is a constant $\lambda_{0} \in(0, \infty)$ such that $\left\{v_{m_{n}, n, \lambda}\right\}_{n \geqq 1}$ is tight for each $\lambda \in\left[0, \lambda_{0}\right]$, provided $\lim \sup _{n \rightarrow \infty} n^{-1} m_{n}^{2}(\log n)^{5 / 2}<\infty$. Let

$$
\begin{gathered}
\mathscr{V}(\lambda)=\left\{\mu_{\lambda}: v_{m_{k_{n}}, k_{n}, \lambda} \text { converges weakly to } \mu_{\lambda} \text { as } n \rightarrow \infty\right. \\
\text { for some } \left.\left\{k_{n}\right\}_{n \geqq 1} \text { with } \lim _{n \rightarrow \infty} k_{n}=\infty\right\} .
\end{gathered}
$$

By considering the expansion of moments of $\mu_{\lambda}$ in powers of $\lambda$ one can see that $\mu_{\lambda}$ is different from the original polymer measure $v_{\lambda}$ in two dimensions for all $\mu_{\lambda} \in \mathscr{V}(\lambda)$, if $\lim _{n \rightarrow \infty} n^{-1} m_{n}^{2}(\log n)^{3}=\infty$.

We shall now give another approach to the problem of showing that $\mu_{\lambda} \neq v_{\lambda}$, from which we can also see that $\mu_{\lambda}, \lambda>0$, should be singular with respect to the Wiener measure $\mu$.

We first prove two lemmas. In this section, we always set $m=m_{n}$.

Lemma 5.1. For any given $\lambda \in[0, \infty)$ there is a constant $c_{1} \in(0, \infty)$ such that

$$
E \exp \left(8 \lambda^{2} n^{-2} m^{2} \sum_{k=1}^{n} \gamma I_{\left\{X_{k} \in Z_{m}^{2}\right\}}\right) \leqq \exp \left(c_{1} \lambda^{2} n^{-1} \gamma\right) \text {. }
$$

Proof. By definition we know that $\gamma=O(1)\left(m^{2}(\log n)^{3}+1\right)$. Without loss of generality, we may assume $\lim _{n \rightarrow \infty} n^{-1} \gamma=\infty$. For any given $\varepsilon \in(0,1)$ we set $\xi(n)=\left[\varepsilon n^{2} \gamma^{-1}\right]$. It is easy to show that

$$
m^{2} E_{x} I_{\left\{X_{k} \in Z_{m}^{2}\right\}} \leqq O(1)\left(m^{2} k^{-1}+1\right), \quad \forall x \in Z^{2},
$$

and for some constant $c_{2} \in(0, \infty)$,

$$
E_{x}\left(n^{-2} m^{2} \sum_{k=1}^{\xi(n)} \gamma I_{\left\{X_{k} \in Z_{m}^{2}\right\}}\right)^{i} \leqq i ! n^{-2 i} m^{2 i} \gamma^{i} E \sum_{1 \leqq k_{1} \leqq \cdots \leqq k_{i} \leqq \xi(n)} I_{\left\{X_{k_{1}}, \ldots, X_{k_{i}} \in Z_{m}^{2}\right\}} \leqq \varepsilon^{2 i} c_{2}^{i} i !,
$$

which implies that

$$
E_{x} \exp \left(8 \lambda^{2} n^{-2} m^{2} \sum_{k=1}^{\xi(n)} \gamma I_{\left\{X_{k} \in Z_{m}^{2}\right\}}\right) \leqq \sum_{i=0}^{\infty}\left(8 \lambda^{2} \varepsilon c_{2}\right)^{i}<1+c_{3} \lambda^{2}
$$

for some constant $c_{3} \in(0, \infty)$, if $\varepsilon \in(0,1)$ is small enough. By the Markov property we have

$$
\begin{aligned}
& E \exp \left(8 \lambda^{2} n^{-2} m^{2} \sum_{k=1}^{n} \gamma I_{\left\{X_{k} \in Z_{m}^{2}\right\}}\right)=E \exp \left(8 \lambda^{2} n^{-2} m^{2} \sum_{k=1}^{n-\xi(n)} \gamma I_{\left\{X_{k} \in Z_{m}^{2}\right\}}\right) \\
& \quad \times E_{X_{n}-\xi(n)} \exp \left(8 \lambda^{2} n^{-2} m^{2} \sum_{k=1}^{\xi(n)} \gamma I_{\left\{X_{k} \in Z_{m}^{2}\right\}}\right) \\
& \leqq\left(1+c_{3} \lambda^{2}\right) E \exp \left(8 \lambda^{2} n^{-2} m^{2} \sum_{k=1}^{n-\xi(n)} \gamma I_{\left\{X_{k} \in Z_{m}^{2}\right\}}\right) \\
& \leqq O(1)\left(1+c_{3} \lambda^{2}\right)^{\frac{n-\xi(n)}{\xi(n)}} \leqq O(1) \exp \left(c_{4} \lambda^{2} n^{-1} m^{2}(\log n)^{3}\right)
\end{aligned}
$$

for some constant $c_{4} \in(0, \infty)$, if $\varepsilon \in(0,1)$ is chosen to be small enough. This completes the proof of Lemma 5.1 . 
Lemma 5.2. If $\lim \sup _{n \rightarrow \infty} n^{-1} m^{2}(\log n)^{5 / 2}<\infty$, then

$$
E\left|n^{-1} m^{2} \sum_{k=1}^{n} I_{\left\{X_{k} \in Z_{m}^{2}\right\}}-1\right|^{2} \leqq O(1) n^{-1} m^{2}(\log n) .
$$

Proof. In fact, we can show that (see e.g. Lemma 6.3 below)

$$
\begin{aligned}
& E\left(n^{-1} m^{2} \sum_{k=1}^{n} I_{\left\{X_{k} \in Z_{m}^{2}\right\}}-1\right)=n^{-1} \sum_{k=1}^{n}\left(m^{2} P\left(X_{k}=0\right)-P\left(X_{k} \in S_{n}(0)\right)\right) \\
& \quad+n^{-1} \sum_{i=1}^{n} \sum_{x \in Z_{m}^{2} \backslash\{0\}} \sum_{y \in S_{n}(x)}\left(P\left(X_{k}=x\right)-P\left(X_{k}=y\right)\right)=O(1) n^{-1} \sum_{k=1}^{n} m^{2} k^{-1} .
\end{aligned}
$$

Moreover, we have

$$
\begin{aligned}
& E\left(n^{-1} m^{2} \sum_{k=1}^{n} I_{\left\{X_{k} \in Z_{m}^{2}\right\}}\right)^{2}-1=n^{-2} m^{4} E \sum_{k=1}^{n} I_{\left\{X_{k} \in Z_{m}^{2}\right\}} \\
& \quad+2 n^{-2} E \sum_{k_{1}=1}^{n} m^{4} I_{\left\{X_{k_{1}} \in Z_{m}^{2}\right\}} \sum_{k_{2}=k_{1}+1} I_{\left\{X_{k_{2}} \in Z_{m}^{2}\right\}}-1 \\
& =O(1) n^{-1} m^{2}+2 n^{-2} E \sum_{k_{1}=1}^{n} m^{2} I_{\left\{X_{k_{1}} \in Z_{m}^{2}\right\}} E \sum_{k_{2}=1}^{n-k_{1}}\left(m^{2} I_{\left\{X_{k_{2}} \in Z_{m}^{2}\right\}}-1\right) \\
& \quad+2 n^{-2} E \sum_{k_{1}=1}^{n} m^{2} I_{\left\{X_{k_{1}} \in Z_{m}^{2}\right\}}\left(n-k_{1}\right)-2 n^{-2} \sum_{k_{1}=1}^{n} \sum_{k_{2}=1}^{n-k_{1}} 1 \\
& =O(1) n^{-1} m^{2} \log n .
\end{aligned}
$$

Hence,

$$
\begin{aligned}
E\left(n^{-1} m^{2} \sum_{k=1}^{n} I_{\left\{X_{k} \in Z_{m}^{2}\right\}}-1\right)^{2}= & E\left(n^{-1} m^{2} \sum_{k=1}^{n} I_{\left\{X_{k} \in Z_{m}^{2}\right\}}\right)^{2}-1 \\
& +2\left(1-E n^{-1} m^{2} \sum_{k=1}^{n} I_{\left\{X_{k} \in Z_{m}^{2}\right\}}\right) \\
\leqq & O(1) n^{-1} m^{2} \log n,
\end{aligned}
$$

which implies the desired result.

Note. It seems that the estimate given in Lemma 5.2 cannot be improved. Lemma 5.1 and Lemma 5.2 will be used again in the proof of Theorem 2.2 in Sect. 6.

For convenience, from now on we always assume in this section that $\lambda>0$, $\lim _{n \rightarrow \infty} n^{-1} m^{2}(\log n)^{3}=\infty$ and $\lim _{n \rightarrow \infty} n^{-1} m^{2}(\log n)^{5 / 2}=0$.

For $\omega, \omega^{\prime} \in C_{0}\left([0, T] \rightarrow R^{2}\right)$, we define

$$
\rho\left(\omega, \omega^{\prime}\right)=\max _{0 \leqq t \leqq T}\left|x(t, \omega)-x\left(t, \omega^{\prime}\right)\right|,
$$

where $x(t, \omega)$ is the position of $\omega$ in $R^{2}$. Then $\tilde{\Omega}_{T}=C_{0}\left([0, T] \rightarrow R^{2}\right)$ is a Polish space (i.e. a complete separable metric space). Using the embedding theorem (see 
e.g. [2, Theorem 2.1]), for any $n \geqq 1$ we may assume that there are non-negative random vectors $\tau_{1} \leqq \tau_{2} \leqq \cdots$ in $R^{2}$ on $\left(\tilde{\Omega}_{T}, \mathscr{B}, \mu\right)$ such that

(i) $\tau_{1}, \tau_{2}-\tau_{1}, \tau_{3}-\tau_{2}, \ldots$ are independent, identically distributed and finite,

(ii) $\tilde{E} \tau_{1}=\left(\frac{1}{n}, \frac{1}{n}\right) \in R^{2}$, where $\tilde{E}$ is the expectation with respect to $\mu$,

(iii) the random vectors $x\left(\tau_{1}, \cdot\right), x\left(\tau_{2}, \cdot\right)-x\left(\tau_{1}, \cdot\right), \ldots$ under the measure $\mu$ are independent and identically distributed, and their distribution is the same as that of $n^{-1 / 2} X_{1}$ under $P$, where $x(u, \cdot)=\left(x_{1}\left(u_{1}, \cdot\right), x_{2}\left(u_{2}, \cdot\right)\right)$, provided $u=\left(u_{1}, u_{2}\right)$ and $x=\left(x_{1}, x_{2}\right)$.

From (iii) we can see that $\left\{n^{-1 / 2} X_{1}, \ldots, n^{-1 / 2} X_{k}\right\}$ and $\left\{x\left(\tau_{1}\right), \ldots, x\left(\tau_{k}\right)\right\}$ have the same distribution. We will prove that $\mu_{\lambda} \neq v_{\lambda}$ for all $\mu_{\lambda} \in \mathscr{V}(\lambda)$. For this purpose, we may assume

$$
v_{m, n, \lambda} \stackrel{\mathscr{D}}{\rightarrow} \mu_{\lambda}, \quad n \rightarrow \infty
$$

Using the embedding theorem given before one can see that $v_{m, n, \lambda}$ can be thought of as a probability measure on $\tilde{\Omega}_{T}$ for $T>1$. Thus, by [27, Theorem 1.1.1] we know that

$$
\lim _{n \rightarrow \infty} v_{m, n, \lambda}(B)=\mu_{\lambda}(B)
$$

for any $B \in \mathscr{B}$ with $\mu_{\lambda}(\partial B)=0$, where $\mathscr{B}$ is the Borel $\sigma$-field. We remark that $v_{\lambda}$ is equivalent to the Wiener measure $\mu$. To get our desired result, it suffices to prove that there is a sequence of sets $\left\{D_{n}\right\}_{n \geqq 1} \subset \tilde{\Omega}_{T}$ such that

$$
\lim _{n \rightarrow \infty} \mu\left(D_{n}\right)=0 ; \quad \limsup _{n \rightarrow \infty} \mu_{\lambda}\left(D_{n}\right)>0,
$$

or

$$
\liminf _{n \rightarrow \infty} \mu\left(D_{n}\right)>0 ; \quad \liminf _{n \rightarrow \infty} \mu_{\lambda}\left(D_{n}\right)=0 .
$$

We set

$$
\begin{aligned}
\zeta_{1}(n)= & n^{-1} m^{2} \sum_{i=1}^{n} \sum_{j=i+1}^{n} I_{\left\{x\left(\tau_{i}, \omega\right)=x\left(\tau_{j}, \omega\right) \in n^{-1 / 2} Z_{m}^{2}\right\}} \\
& -n^{-1} m^{2} \sum_{i=1}^{n} \beta I_{\left\{x\left(\tau_{i}, \omega\right) \in n^{-1 / 2} Z_{m}^{2}\right\}}, \\
\zeta_{2}(n)= & n^{-2} m^{2} \gamma \sum_{i=1}^{n} I_{\left\{x\left(\tau_{i}, \omega\right) \in n^{-1 / 2} Z_{m}^{2}\right\}},
\end{aligned}
$$

where $n^{-1 / 2} S=\left\{n^{-1 / 2} x: x \in S\right\}$. We also set

$$
\begin{aligned}
& B_{n}=\left\{\omega \in \tilde{\Omega}_{1+n^{-1 / 4}}: \tau_{n} \leqq\left(1+\frac{1}{n^{1 / 4}}, 1+\frac{1}{n^{1 / 4}}\right),\left|\zeta_{1}(n)\right| \geqq \frac{1}{2} \zeta_{2}(n)\right\}, \\
& P_{n}=P\left(\left|\frac{1}{2} S_{m, n}-n^{-1} m^{2} \sum_{i=1}^{n} \beta I_{\left\{X_{i} \in Z_{m}^{2}\right\}}\right| \geqq \frac{1}{2} n^{-2} m^{2} \gamma \sum_{i=1}^{n} I_{\left\{X_{i} \in Z_{m}^{2}\right\}}\right) .
\end{aligned}
$$


By Lemma 5.2 we can show that

$$
\begin{aligned}
P_{n} \leqq & P\left(\left|n^{-1} \sum_{i=1}^{n} m^{2} I_{\left\{X_{i} \in Z_{m}^{2}\right\}}-1\right| \geqq(\log n)^{-5 / 4}\right) \\
& +P\left(\left|\frac{1}{2} S_{m, n}-\frac{1}{2} E S_{m, n}\right| \geqq \frac{1}{2} n^{-1} \gamma-O(1)(\log n)^{-1 / 4}\right) \\
\leqq & O(1)(\log n)^{-1 / 4}+O(1) n^{2} \gamma^{-2} \operatorname{Var}\left(S_{m, n}\right) \\
\leqq & O(1)\left((\log n)^{-1 / 4}+\operatorname{Var}^{-1}\left(S_{m, n}\right)\right)
\end{aligned}
$$

goes to zero as $n \rightarrow \infty$, provided $\lim _{n \rightarrow \infty} n^{-1} m^{2}(\log n)^{3}=\infty$. Using the above estimate we can show that

$$
\mu\left(B_{n}\right) \leqq P_{n} \leqq O(1)\left((\log n)^{-1 / 4}+\operatorname{Var}^{-1}\left(S_{m, n}\right)\right)
$$

goes to zero as $n \rightarrow \infty$. Hence, there is a subsequence $\left\{n_{k}\right\}_{k \geqq 1}$ such that

$$
\sum_{k=1}^{\infty} \mu\left(B_{n_{k}}\right)<\infty
$$

which implies that

$$
\lim _{l \rightarrow \infty} \mu\left(A_{l}\right)=1
$$

If we make the plausible assumption that this implies

$$
\lim _{l \rightarrow \infty} \mu\left(\partial A_{l}\right)<1
$$

where $A_{l}=\cap_{k=l}^{\infty} B_{n_{k}}^{c}$, then we can proceed as follows. If $\lim \sup _{l \rightarrow \infty} \mu_{\lambda}\left(\partial A_{l}\right)=1$, then $(5.1)^{\prime}$ holds by setting $\left\{D_{l}\right\}=\left\{\left(\partial A_{l}\right)^{c}\right\}$. We now suppose lim $\sup _{l \rightarrow \infty} \mu_{\lambda}\left(\partial A_{l}\right)$ $<1$. Thus, for any given $\varepsilon \in(0,1)$ there is a constant $l_{0} \geqq 1$ such that

$$
\mu_{\lambda}\left(\partial A_{l}\right) \leqq 1-\varepsilon, \quad \forall l \geqq l_{0} .
$$

From the proof of $[27$, Theorem 1.1.1] we can see that

$$
\mu_{\lambda}\left(A_{l}\right) \leqq \liminf _{n \rightarrow \infty} v_{m, n, \lambda}\left(A_{l}\right)+1-\varepsilon, \quad \forall l \geqq l_{0} .
$$

It is easy to show that

$$
\tilde{E}\left|\tau_{n}-(1,1)\right|^{2} \leqq O(1) n^{-1}
$$

which implies

$$
\mu\left(\tau_{n} \geqq\left(1+n^{-1 / 4}, 1+n^{-1 / 4}\right)\right) \leqq O(1) n^{-1 / 2} .
$$

We remark that $\lim _{n \rightarrow \infty} n^{-1} \gamma(\log n)^{-1 / 2}=0$ and $\lim _{n \rightarrow \infty} n^{-1} \gamma=\infty$. Using these properties we can show that

$$
n^{-2} \gamma m^{2} \sum_{i=1}^{n} I_{\left\{X_{i} \in Z_{m}^{2}\right\}} \stackrel{P}{\rightarrow} \infty, \quad n \rightarrow \infty,
$$

and by Lemma 5.1

$$
E \exp \left(16 \lambda^{2} n^{-2} \gamma m^{2} \sum_{i=1}^{n} I_{\left\{X_{i} \in Z_{m}^{2}\right\}}\right) \leqq \exp \left(c_{5} \lambda^{2} n^{-1} \gamma\right) \leqq O(1) n^{1 / 8}
$$


for some constant $c_{5} \in(0, \infty)$. Thus, by Proposition 4.1 and the Fatou lemma we can show for $l \leqq n$ that

$$
\begin{aligned}
& v_{m, n, \lambda}\left(A_{l}\right) \leqq v_{m, n, \lambda}\left(B_{n}^{c}\right) \\
& \leqq v_{m, n, \lambda}\left(\tau_{n} \geqq\left(1+n^{-1 / 4}, 1+n^{-1 / 4}\right)\right)+O(1) \tilde{E} \exp \left(-2 \lambda^{2} \zeta_{2}(n)\right) \\
& \leqq O(1) n^{-1 / 4} \tilde{E}^{1 / 2} \exp \left(-4 \lambda \zeta_{1}(n)-8 \lambda^{2} \zeta_{2}(n)\right)+O(1) \tilde{E} \exp \left(-2 \lambda^{2} \zeta_{2}(n)\right) \\
& \leqq O(1) n^{-1 / 4} E^{1 / 4} \exp \left(16 \lambda^{2} n^{-2} \gamma m^{2} \sum_{i=1}^{n} I_{\left\{X_{i} \in Z_{m}^{2}\right\}}\right) \\
&+O(1) E \exp \left(-2 \lambda^{2} n^{-2} \gamma m^{2} \sum_{i=1}^{n} I_{\left\{X_{i} \in Z_{m}^{2}\right\}}\right)
\end{aligned}
$$

goes to zero as $n \rightarrow \infty$ if $\lambda>0$. From this and (5.2) we obtain that

$$
\varlimsup_{l \rightarrow \infty} \mu_{\lambda}\left(A_{l}\right) \leqq 1-\varepsilon .
$$

This proves $(5.1)$ by choosing $\left\{D_{l}\right\}=\left\{A_{l}^{c}\right\}$.

\section{Proof of Theorem 2.2}

In this section we first assume $\lim \sup _{n \rightarrow \infty} n^{-1} m_{n}^{2}(\log n)^{5 / 2}<\infty$ and set $m=m_{n}$. We first prove the following proposition.

Proposition 6.1. There are constants $\lambda_{0} \in(0, \infty)$ and $c_{1} \in(0, \infty)$ such that

$$
\begin{aligned}
& E \exp \left(-\lambda m^{2} \beta n^{-1} \sum_{i=1}^{n} I_{\left\{X_{i} \in Z_{m}^{2}\right\}}+\lambda \beta\right) \\
& \quad \leqq O(1) \exp \left(c_{1} \lambda n^{-1} m^{2}(\log n)^{3}\right), \quad \lambda \in\left[0, \lambda_{0}\right] .
\end{aligned}
$$

In particular, if $\lim \sup _{n \rightarrow \infty} n^{-1} m^{2}(\log n)^{3}<\infty$, then for any given $\lambda \in[0, \infty)$ there is a constant $c_{1} \in(0,1)$ such that

$$
c_{1} \leqq E \exp \left(-\lambda m^{2} \beta n^{-1} \sum_{i=1}^{n} I_{\left\{X_{i} \in Z_{m}^{2}\right\}}+\lambda \beta\right) \leqq c_{1}^{-1} .
$$

We will use the approach presented in the proof of [5, Proposition 3.4] to prove Proposition 6.1. We set

$$
\begin{gathered}
I_{k, l}^{m, \lambda}=\lambda m^{2} \beta n^{-1} \sum_{i=k}^{l} I_{\left\{X_{i} \in Z_{m}^{2}\right\}}-\lambda n^{-1} \sum_{i=k}^{l} \beta, \\
q_{k, l}^{m, \lambda}=E \exp \left(-I_{k, l}^{m, \lambda}\right),
\end{gathered}
$$

and $q_{l}^{m, \lambda}=q_{1, l}^{m, \lambda}$. We will also drop $m, \lambda$ from the above notations. We begin with several lemmas.

Lemma 6.2. Suppose that $0<l_{2}-l_{1} \leqq m^{2}$ and $l_{1} \geqq\left[n(\log n)^{-1}\right]$. Then

$$
\left|E \exp \left(-I_{1, l_{1}}\right)\left(\xi_{1}-\xi_{2}\right)\right| \leqq O(1) \lambda q_{l_{1}} n^{-1} m^{2} \log n \log \log n,
$$


where

$$
\xi_{1}=\lambda \beta m^{2} n^{-1} \sum_{k=l_{1}+1}^{l_{2}} I_{\left\{X_{k} \in Z_{m}^{2}\right\}}, \quad \xi_{2}=\lambda n^{-1} \sum_{k=l_{1}+1}^{l_{2}} \beta .
$$

Proof. Without loss of generality, we may assume $n^{-1} m^{2} \geqq O(1)(\log n)^{-K}$ for some constant $K \geqq 3$. Clearly, we have

$$
\begin{aligned}
E \exp \left(-I_{1, l_{1}}\right) \xi_{2} & \leqq \lambda \beta n^{-1}\left(l_{2}-l_{1}-1\right) E \exp \left(-I_{1, l_{1}}\right) \\
& \leqq O(1) \lambda q_{l_{1}} n^{-1} m^{2} \log n
\end{aligned}
$$

By definition we know that

$$
q_{l_{1}} \leqq q_{l_{1}-i} \exp \left(\lambda \beta n^{-1} i\right) \leqq O(1) q_{l_{1}-i}
$$

if $i \leqq O(1) n(\log n)^{-1}$. On the other hand, we have

$$
\begin{aligned}
& E_{X_{l_{1}-i}} \sum_{k=1}^{i} \lambda \beta n^{-1} m^{2} I_{\left\{X_{k} \in Z_{m}^{2}\right\}} \leqq O(1) E_{0} \sum_{k=1}^{i} \lambda \beta n^{-1} m^{2} I_{\left\{X_{k} \in Z_{m}^{2}\right\}} \\
& \leqq O(1), \quad i \leqq O(1) n(\log n)^{-1}
\end{aligned}
$$

which implies that

$$
\begin{aligned}
q_{l_{1}} & =E \exp \left(-I_{1, l_{1}-i}\right) E_{X_{l_{1}-i}} \exp \left(-I_{1, i}\right) \geqq E \exp \left(-I_{1, l_{1}-i}\right) \exp \left(-E_{X_{l_{1}-i}} I_{1, i}\right) \\
& \geqq O(1) q_{l_{1}-i} .
\end{aligned}
$$

Remark that

$$
\begin{aligned}
& \lambda \beta n^{-1} \sum_{k=l_{1}+1}^{l_{2}} E m^{2} I_{\left\{X_{k} \in Z_{m}^{2}\right\}} \exp \left(-I_{1, l_{1}}\right) \\
& \quad \leqq O(1) \lambda \beta n^{-1} \sum_{k=l_{1}+1}^{l_{2}} E \exp \left(-I_{1, l_{1}-i}\right) E_{X_{l_{1}-i}} E_{X_{i}} m^{2} I_{\left\{X_{k-l_{1}} \in Z_{m}^{2}\right\}} \\
& \quad \leqq O(1) \lambda \beta n^{-1} q_{l_{1}-i} \sum_{k=1}^{l_{2}-l_{1}} \max _{x \in Z^{2}} E_{x} E_{X_{i}} m^{2} I_{\left\{X_{k} \in Z_{m}^{2}\right\}},
\end{aligned}
$$

and

$$
\left.P_{x}\left(\operatorname{dist}\left(X_{i}, Z_{m}^{2}\right) \leqq m(\log n)^{-2}\right)\right) \leqq O(1)(\log n)^{-2}
$$

We choose $i=\left[n(\log n)^{-1} / 2\right]$. We also remark that

$$
\begin{aligned}
\lambda \beta n^{-1} \sum_{k=1}^{l_{2}-l_{1}} E_{X_{i}} m^{2} I_{\left\{X_{k} \in Z_{m}^{2}\right\}} & \leqq O(1) \lambda \beta n^{-1}\left(m^{2} \sum_{k=1}^{l_{2}-l_{1}} k^{-1}+l_{2}-l_{1}\right) \\
& \leqq O(1) \lambda n^{-1} m^{2}(\log n)^{2}
\end{aligned}
$$


Thus, we can show that $E \exp \left(-I_{1, l_{1}}\right) \xi_{1}$ is less than

$$
\begin{aligned}
O(1) & \lambda n^{-1}\left(m^{2} q_{l_{1}-i}+\beta \sum_{k=1}^{l_{2}-l_{1}} \max _{x \in Z^{2}} E_{x} I_{\left\{\operatorname{dist}\left(X_{i}, Z_{m}^{2}\right) \geqq m(\log n)^{-2}\right\}}\right. \\
& \left.\times E_{X_{i}-\eta\left(X_{i}\right)} m^{2} I_{\left\{X_{k} \in Z_{m}^{2}\right\}} q_{l_{1}-i}\right) \\
\leqq & O(1) \lambda n^{-1} q_{l_{1}-i}\left(m^{2}+\beta \sum_{k=1}^{l_{2}-l_{1}} \operatorname{dist}\left(x, Z_{m}^{2}\right) \geqq m(\log n)^{-2}\right. \\
\leqq & \left.\max _{x-\eta(x)} m^{2} I_{\left\{X_{k} \in Z_{m}^{2}\right\}}\right) \\
& \left.+\sum_{k=\left[m^{2}(\log n)^{-8}\right]}^{l_{2}-l_{1}} \max _{x \in Z^{2}} E_{x} m^{2} I_{\left\{X_{k} \in Z_{m}^{2}\right\}}\right) \\
\leqq & O(1) \lambda n^{-1} q_{l_{1}-i}\left(m^{2}+\beta \sum_{k=1}^{\left[m^{2}(\log n)^{-8}\right]} m^{2} P_{x-\eta(x)}\left(X_{k} \in Z_{m}^{2}\right)\right. \\
\leqq & O(1) \lambda q_{l_{1}-i} n^{-1}\left(m^{2}+m^{2} \log \left(x, Z_{m}^{2}\right) \geqq m(\log n)^{-2} \log \log n\right) \\
\leqq & O(1) \lambda n^{-1} m^{2} q_{l_{1}} \log n \log \log n,
\end{aligned}
$$

which proves the desired result.

Lemma 6.3. Suppose that there is a constant $c_{2} \in(0, \infty)$ such that $1 \leqq l_{2}-l_{1} \leqq$ $c_{2} n(\log n)^{-3 / 2}$ and $l_{1} \geqq\left[n(\log n)^{-1}\right]$. Then, for any given $\lambda \in[0, \infty)$ there is $a$ constant $c_{3} \in(0, \infty)$ such that

$$
\left|E \exp \left(-I_{1, l_{1}}\right)\left(\xi_{1}-\xi_{2}\right)\right| \leqq c_{3} n^{-1} m^{2} q_{l_{1}} \log n \log \log n
$$

Proof. We will use the approach given in [5, Sect.3] to prove (6.1). By Lemma 6.2 we know that $(6.1)$ holds if $l_{2}-l_{1} \leqq m^{2}$. We now assume $l_{2}-l_{1} \geqq$ $m^{2}+1$. By the Markov property we have

$$
\begin{aligned}
E \exp \left(-I_{1, l}\right)\left(\xi_{1}-\xi_{2}\right)=\lambda n^{-1} \beta \sum_{k=l_{1}+1}^{l_{2}} E \exp \left(-I_{1, l_{1}}\right)\left(m^{2} I_{\left\{X_{k} \in Z_{m}^{2}\right\}}-1\right) \\
=\lambda n^{-1} \beta \sum_{k=1}^{l_{2}-l_{1}} E \exp \left(-I_{1, l_{1}}\right) E_{X_{l_{1}}}\left(m^{2} I_{\left\{X_{k} \in Z_{m}^{2}\right\}}-1\right) \\
=\lambda n^{-1} \beta \sum_{k=1}^{m^{2}} E \exp \left(-I_{1, l_{1}}\right) E_{X_{l_{1}}}\left(m^{2} I_{\left\{X_{k} \in Z_{m}^{2}\right\}}-1\right) \\
\quad+\lambda n^{-1} \beta \sum_{k=m^{2}+1}^{l_{2}-l_{1}} E \exp \left(-I_{1, l_{1}}\right) E_{X_{l_{1}}}\left(m^{2} I_{\left\{X_{k} \in Z_{m}^{2}\right\}}-1\right) \\
=O\left(\lambda n^{-1} m^{2} q_{l_{1}} \log n \log \log n\right)+\lambda n^{-1} \beta E \exp \left(-I_{1, l_{1}}\right) \\
\quad \times \sum_{k=m^{2}+1}^{l_{2}-l_{1}} E_{X_{l_{1}}}\left(m^{2} I_{\left\{X_{k} \in Z_{m}^{2}\right\}}-1\right) .
\end{aligned}
$$


By the symmetry property of $\left\{X_{n}\right\}$ we know that

$$
E \exp \left(-I_{1, l_{1}}\right) \sum_{z \in S_{n}(0)} z \cdot\left(X_{k+l_{1}}-X_{l_{1}}\right) I_{\left\{X_{k+l_{1}} \in Z_{m}^{2} \backslash\{0\}\right\}}=0
$$

which implies that

$$
E \exp \left(-I_{1, l_{1}}\right) \sum_{\left.y \in Z_{m}^{2} \backslash\{0\}\right\}} \sum_{z \in S_{n}(0)} z \cdot\left(y-X_{l_{1}}\right) p_{k}\left(y-X_{l_{1}}\right)=0,
$$

where $S_{n}(x)$ was defined in Sect. 3, and $u \cdot v=u_{1} v_{1}+u_{2} v_{2}$, provided $u=\left(u_{1}, u_{2}\right)$ and $v=\left(v_{1}, v_{2}\right)$.

It is easy to show that

$$
\begin{aligned}
& n^{-1} \beta E \exp \left(-I_{1, l_{1}}\right) \sum_{k=m^{2}+1}^{l_{2}-l_{1}}\left(m^{2} p_{k}(-x)+\sum_{y \in S_{n}(0)} p_{k}(y-x)\right) \\
& \leqq O(1) q_{l_{1}} n^{-1} \beta m^{2} \log \frac{l_{2}-l_{1}}{m^{2}+1} \leqq O(1) q_{l_{1}} n^{-1} m^{2} \log \log n
\end{aligned}
$$

By [20, Prop. 1.2.5] we know that if $\alpha<2 / 3,|x| \leqq k^{\alpha}$ and $p_{k}(x)>0$

$$
p_{k}(x)=\bar{p}(k, x)\left(1+O\left(k^{3 \alpha-2}\right)\right) .
$$

For convenience, when we estimate the difference: $p_{t}(x)-p_{t}(y)$, we always assume $p_{t}(x) p_{t}(y)>0$, or $p_{t}(x)=0$ and $p_{t}(y)=0$. Otherwise, we may consider $p_{t}(x)$ and $p_{t+1}(y)$, or $p_{t+1}(x)$ and $p_{t}(y)$. Thus, there is a constant $\delta \in(0,1)$ such that

$$
\begin{aligned}
& n^{-1} \beta E \exp \left(-I_{1, l_{1}}\right) \sum_{k=m^{2}+1}^{l_{2}-l_{1}} E_{X_{l_{1}}}\left(m^{2} I_{\left\{X_{k} \in Z_{m}^{2}\right\}}-1\right) \\
& =O\left(n^{-1} \beta m^{2} \sum_{k=m^{2}+1}^{l_{2}-l_{1}} k^{-1} q_{l_{1}}\right)+n^{-1} \beta E \exp \left(-I_{1, l_{1}}\right) \\
& \times \sum_{k=m^{2}+1}^{l_{2}-l_{1}}\left[m^{2} \sum_{y \in Z_{m}^{2} \backslash\{0\}} p_{k}\left(y-X_{l_{1}}\right)-\sum_{y \in Z \backslash S_{n}(0)} p_{k}\left(y-X_{l_{1}}\right)\right] \\
& =O\left(n^{-1} \beta \sum_{k=m^{2}+1}^{l_{2}-l_{1}}\left(m^{2} k^{-1}+k^{-\delta}\right)\right) q_{l_{1}} \\
& +\frac{2}{\pi} n^{-1} \beta \sum_{k=m^{2}+1}^{l_{2}-l_{1}} \sum_{y \in Z_{m}^{2} \backslash\{0\}} \sum_{z \in S_{n}(0)} E \exp \left(-I_{1, l_{1}}\right) \frac{1}{k} \\
& \times \exp \left(-\frac{\left|y-X_{l_{1}}\right|^{2}}{k}\right)\left[1-\exp \left(-\frac{\left|y-X_{l_{1}}+z\right|^{2}-\left|y-X_{l_{1}}\right|^{2}}{k}\right)\right] \\
& =O\left(n^{-1} m^{2} \log n \log \log n\right) q_{l_{1}}+\frac{2}{\pi} n^{-1} \beta \sum_{k=m^{2}+1}^{l_{2}-l_{1}} \sum_{y \in Z_{m}^{2} \backslash\{0\}} E \exp \left(-I_{1, l_{1}}\right) \\
& \times p_{k}\left(y-X_{l_{1}}\right)\left\{I _ { \{ | y - X _ { l _ { 1 } } | ^ { 2 } \leqq M k \} } \sum _ { z \in S _ { n } ( 0 ) } \left[\frac{2 z \cdot\left(y-X_{l_{1}}\right)+|z|^{2}}{k}\right.\right.
\end{aligned}
$$




$$
\begin{aligned}
& \left.+O\left(\frac{2 z \cdot\left(y-X_{l_{1}}\right)+|z|^{2}}{k}\right)^{2}\right]+I_{\left\{\left|y-X_{l_{1}}\right|^{2}>M k\right\}} \\
& \left.\times \sum_{z \in S_{n}(0)} \sum_{i=1}^{\infty} \frac{1}{i !} \frac{\left(2 z \cdot\left(y-X_{l_{1}}\right)+|z|^{2}\right)^{i}}{k^{i}}\right\} \\
& =O\left(n^{-1} m^{2} \log n \log \log n\right) q_{l_{1}}+\frac{2}{\pi} n^{-1} \beta \sum_{k=m^{2}+1}^{l_{2}-l_{1}} \sum_{y \in Z_{m}^{2} \backslash\{0\}} E \exp \left(-I_{1, l_{1}}\right) \\
& \times p_{k}\left(y-X_{l_{1}}\right)\left\{I_{\left\{\left|y-X_{l_{1}}\right|^{2} \leqq M k\right\}} \sum_{z \in S_{n}(0)}\left[\frac{|z|^{2}}{k}+O\left(\frac{2 z \cdot\left(y-X_{l_{1}}\right)+|z|^{2}}{k}\right)^{2}\right]\right. \\
& \left.+I_{\left\{\left|y-X_{l_{1}}\right|^{2}>M k\right\}} \sum_{z \in S_{n}(0)}\left(\frac{|z|^{2}}{k}+\sum_{i=2}^{\infty} \frac{1}{i !} \frac{\left(2 z \cdot\left(y-X_{l_{1}}\right)+|z|^{2}\right)^{i}}{k^{i}}\right)\right\} \\
& =: O\left(n^{-1} m^{2} \log n \log \log n\right) q_{l_{1}}+U_{1}+U_{2} .
\end{aligned}
$$

Using an argument for $L_{1}^{\prime}$ given in the proof of [5, Lemma 3.3] we can show that $U_{1}$ is less than

$$
\begin{aligned}
& O(1) n^{-1} \beta \sum_{k=m^{2}+1}^{l_{2}-l_{1}} \sum_{y \in Z_{m}^{2} \backslash\{0\}} \frac{m^{4}}{k} E \exp \left(-I_{1, l_{1}}\right) p_{k}\left(y-X_{l_{1}}\right) \\
& \leqq O(1) n^{-1} \beta \sum_{k=m^{2}+1}^{l_{2}-l_{1}}\left(m^{4} k^{-2}+m^{2} k^{-1}\right) q_{l_{1}} \\
& \leqq O(1) q_{l_{1}} n^{-1} m^{2} \log n \log \log n .
\end{aligned}
$$

Using the argument for $L_{1}^{\prime \prime}$ given in the proof of [5, Lemma 3.3], we can show that there is a constant $c_{4} \in(0, \infty)$ such that $U_{2}$ is less than

$$
\begin{aligned}
& O(1) n^{-1} \beta \sum_{k=m^{2}+1}^{l_{2}-l_{1}} \sum_{y \in Z_{m}^{2} \backslash\{0\}} E \exp \left(-I_{1, l_{1}}\right) p_{k}\left(y-X_{l_{1}}\right) \\
& \times \frac{m^{4}}{k} \sum_{i=1}^{\infty} \frac{c_{4}^{i}}{i !} \frac{\left|y-X_{l_{1}}\right|^{i}}{k^{i / 2}} I_{\left\{\left|y-x_{l_{1}}\right|^{2}>M k\right\}} \\
& \leqq \\
& O(1) n^{-1} \beta \sum_{k=m^{2}+1}^{l_{2}-l_{1}} \frac{1}{k} E \exp \left(-I_{1, l_{1}}\right) \sum_{y \in Z_{m}^{2} \backslash\{0\}} \exp \left(-\frac{\left|y-X_{l_{1}}\right|^{2}}{k}\right) \\
& \times \frac{m^{4}}{k} \exp \left(-\frac{c_{4}\left|y-X_{l_{1}}\right|}{k^{1 / 2}}\right) I_{\left\{\left|y-X_{l_{1}}\right|^{2}>M k\right\}} \\
& \leqq O(1) n^{-1} \beta m^{4} \sum_{k=m^{2}+1}^{l_{2}-l_{1}} \frac{1}{k^{2}} E \exp \left(-I_{1, l_{1}}\right) \\
& \times \sum_{y \in Z_{m}^{2} \backslash\{0\}} \exp \left(-\frac{\left|y-X_{l_{1}}\right|^{2}}{2 k}\right) I_{\left\{\left|y-X_{l_{1}}\right|^{2}>M k\right\}} \\
& \leqq O(1) q_{l_{1}} n^{-1} m^{2} \log n \log \log n,
\end{aligned}
$$

if $M \geqq 1$ is chosen to be large enough. Combining the above estimates, we obtain the desired conclusion. 
Lemma 6.4. Suppose that there is a constant $c_{5} \in(0, \infty)$ such that $0<l_{2}-l_{1} \leqq$ $c_{5} n(\log n)^{-3 / 2}$ and $l_{1} \geqq\left[n(\log n)^{-1}\right]$. Then, for any given $\lambda \in[0, \infty)$ there is $a$ constant $c_{6} \in(0, \infty)$

$$
\left|q_{l_{2}}-q_{l_{1}}\right| \leqq c_{6} q_{l_{1}}\left((\log n)^{-3 / 2}+n^{-1} m^{2}(\log n)^{3 / 2}\right) .
$$

Proof. We remark that

$$
1-x+\frac{x^{2}}{2}-\frac{x^{3}}{6} \leqq e^{-x} \leqq 1-x+\frac{x^{2}}{2}, \quad \forall x \geqq 0,
$$

and $e^{x}=1+x+\frac{x^{2}}{2}+O\left(x^{3}\right)$ if $|x| \leqq M$ for some constant $M \in(0, \infty)$. Then, we have

$$
\begin{aligned}
q_{l_{1}}-q_{l_{2}} & =E \exp \left(-I_{1, l_{1}}\right)\left(1-\exp \left(-\xi_{1}+\xi_{2}\right)\right) \\
& \leqq E \exp \left(-I_{1, l_{1}}\right)\left(1-\exp \left(\xi_{2}\right)\left(1-\xi_{1}+\frac{\xi_{1}^{2}}{2}-\frac{\xi_{1}^{3}}{6}\right)\right) \\
& =E \exp \left(-I_{1, l_{1}}\right)\left[\left(\xi_{1}-\xi_{2}\right)+\xi_{1} \xi_{2}-\frac{\xi_{1}^{2}}{2}-\frac{\xi_{2}^{2}}{2}+O\left(\sum_{u+v \geqq 3} \xi_{1}^{u} \xi_{2}^{v}\right)\right] .
\end{aligned}
$$

We first consider $E \exp \left(-I_{1, l_{1}}\right) \xi_{1}$. By the Markov property of $\left\{X_{n}\right\}$ we can show that $E \exp \left(-I_{1, l_{1}}\right) \xi_{1}$ is less than

$$
\begin{aligned}
& \lambda \beta m^{2} n^{-1} \sum_{k=l_{1}+1}^{l_{2}} E \exp \left(-I_{1, l_{1}}\right) I_{\left\{X_{k} \in Z_{m}^{2}\right\}} \\
&=\lambda \beta m^{2} n^{-1} \sum_{k=l_{1}+1}^{l_{2}} E \exp \left(-I_{1, l_{1}}\right) P_{X_{l_{1}}}\left(X_{k-l_{1}} \in Z_{m}^{2}\right) \\
& \leqq \lambda \beta m^{2} n^{-1} \sum_{k=l_{1}+1}^{l_{2}} E \exp \left(-I_{1, l_{1}}\right)\left[P_{X_{l_{1}}-\eta\left(X_{l_{1}}\right)}\left(X_{k-l_{1}}=0\right)\right. \\
&\left.+\sum_{x \in Z_{m}^{2} \backslash\{0\}} P_{X_{l_{1}}-\eta\left(X_{l_{1}}\right)}\left(X_{k-l_{1}}=x\right)\right] \\
& \leqq O(1) \lambda \beta n^{-1} E \exp \left(-I_{1, l_{1}}\right) \sum_{k=l_{1}+1}^{l_{2}}\left(\frac{m^{2}}{k-l_{1}}+1\right) \\
& \leqq O(1) \lambda \beta n^{-1}\left(m^{2} \log \left(l_{2}-l_{1}\right)+l_{2}-l_{1}\right) E \exp \left(-I_{1, l_{1}}\right) \\
& \leqq O(1)\left(n^{-1} m^{2}(\log n)^{2}+(\log n)^{-1 / 2}\right) q_{l_{1}} \\
& \leqq O(1)(\log n)^{-1 / 2} q_{l_{1}},
\end{aligned}
$$

where $\eta(x)$ was defined in Sect. 3. By a similar argument as before we can show that

$$
E \exp \left(-I_{1, l_{1}}\right) \xi^{i} \leqq O(1)(\log n)^{-i / 2} q_{l_{1}}, \quad i=2,3
$$

Remark that

$$
\xi_{2}^{i} \leqq O(1) \lambda(\log n)^{-i / 2}, \quad i=1,2,3
$$


Thus, we know by (6.2) that $q_{l_{1}}-q_{l_{2}}$ is less than

$$
E \exp \left(-I_{1, l_{1}}\right)\left(\left(\xi_{1}-\xi_{2}\right)+\xi_{1} \xi_{2}-\frac{\xi_{1}^{2}}{2}-\frac{\xi_{2}^{2}}{2}\right)+O(1)(\log n)^{-3 / 2} q_{l_{1}} .
$$

Similarly, we can use (6.2) to show that $q_{l_{1}}-q_{l_{2}}$ is greater than

$$
E \exp \left(-I_{1, l_{1}}\right)\left(\left(\xi_{1}-\xi_{2}\right)+\xi_{1} \xi_{2}-\frac{\xi_{1}^{2}}{2}-\frac{\xi_{2}^{2}}{2}\right)-O(1)(\log n)^{-3 / 2} q_{l_{1}} .
$$

By Lemma 6.3 we know that

$$
\left|E \exp \left(-I_{1, l_{1}}\right)\left(\xi_{1}-\xi_{2}\right)\right| \leqq O(1) \lambda q_{l_{1}} n^{-1} m^{2} \log n \log \log n .
$$

We remark that (see the proof of Lemma 5.2 above)

$$
\beta n^{-1} \sum_{k=1}^{l_{2}-l_{1}} E\left(m^{2} I_{\left\{X_{k} \in Z_{m}^{2}\right\}}-1\right)=O\left(n^{-1} m^{2}(\log n)^{2}\right) .
$$

Thus, by a similar argument as in the proof of Lemma 6.3 we can show that

$$
\left|E \exp \left(-I_{1, l_{1}}\right)\left(\xi_{1}^{2}-\xi_{2}^{2}\right)\right| \leqq O(1) \lambda^{2} q_{l_{1}} n^{-1} m^{2}(\log n)^{3 / 2} .
$$

Using this and Lemma 6.3 again we can show that

$$
\left|E \exp \left(-I_{1, l_{1}}\right)\left(\xi_{1} \xi_{2}-\frac{\xi_{1}^{2}}{2}-\frac{\xi_{2}^{2}}{2}\right)\right| \leqq O(1) \lambda^{2} q_{l_{1}} n^{-1} m^{2}(\log n)^{3 / 2} .
$$

Thus, we finally obtain that

$$
\left|q_{l_{1}}-q_{l_{2}}\right| \leqq O(1) q_{l_{1}}\left(\lambda+\lambda^{2}+\lambda^{3}\right)\left(n^{-1} m^{2}(\log n)^{3 / 2}+(\log n)^{-3 / 2}\right),
$$

which implies the desired result.

We now use Lemma 6.4 to prove Proposition 6.1.

Proof of Proposition 6.1. By Lemma 6.4 we know that

$$
q_{l_{2}} \leqq\left(1+c_{6} n^{-1} m^{2}(\log n)^{3 / 2}+(\log n)^{-3 / 2}\right) q_{l_{1}},
$$

if $0<l_{2}-l_{1} \leqq O(1) n(\log n)^{-3 / 2}$ and $l_{1} \geqq\left[n(\log n)^{-1}\right]$. We set $k_{0}=\left[n(\log n)^{-1}\right]$ and choose $k_{0}<k_{1}<\cdots<k_{u_{n}-1}<k_{u_{n}}=n$ such that $u_{n} \leqq 2\left[(\log n)^{3 / 2}\right]$ and

$$
k_{i}-k_{i-1} \leqq\left[n(\log n)^{-3 / 2}\right], \quad i=1, \ldots, u_{n} .
$$

Without loss of generality, we may assume $n^{-1} m^{2} \geqq O(1)(\log n)^{-3}$. Then, there are constants $c_{7}, c_{8} \in(0, \infty)$ such that

$$
\begin{aligned}
q_{n} & \leqq\left(1+c_{7} n^{-1} m^{2}(\log n)^{3 / 2}\right) q_{u_{n}-1} \leqq\left(1+c_{7} n^{-1} m^{2}(\log n)^{3 / 2}\right)^{u_{n}} q_{k_{0}} \\
& \leqq O(1) \exp \left(c_{8} n^{-1} m^{2}\right),
\end{aligned}
$$

which implies the desired result.

We are now in a position to complete the proof of Theorem 2.2.

Proof of Theorem 2.2. (i) Let

$$
\bar{S}_{m, n}^{\prime \prime}(\lambda)=\hat{S}_{m, n}(\lambda)+4 \lambda^{2} n^{-1} \gamma .
$$


We first remark that the condition $\lim _{n \rightarrow \infty} n^{-1} \gamma / \log \log n=0$ is equivalent to the condition $\lim _{n \rightarrow \infty} n^{-1} m^{2}(\log n)^{3} / \log \log n=0$. By Lemma 5.1, Proposition 4.1 and the Hölder inequality we can show that

$$
\begin{gathered}
E \exp \left(-2 \bar{S}_{m, n}(\lambda)\right)+E \exp \left(-2 \bar{S}_{m, n}^{\prime \prime}(\lambda)\right) \\
\leqq \exp \left(c_{9} \lambda n^{-1} \gamma\right) \leqq O(1)(\log n)^{\delta_{n}}
\end{gathered}
$$

for some constant $c_{9} \in(0, \infty)$, where $\delta_{n} \in(0, \infty)$ satisfies $\lim _{n \rightarrow \infty} \delta_{n}=0$. Thus, by Proposition 4.1, Lemma 5.1 and Lemma 5.2 we can show that

$$
\begin{aligned}
&\left|E \exp \left(-\bar{S}_{m, n}^{\prime \prime}(\lambda)\right)-E \exp \left(-\bar{S}_{m, n}(\lambda)\right)\right| \\
& \leqq E \exp \left(-\bar{S}_{m, n}(\lambda)\right)\left|1-\exp \left(4 \lambda^{2} n^{-1} \gamma \sum_{i=1}^{n}\left(m^{2} I_{\left\{X_{i} \in Z_{m}^{2}\right\}}-1\right)\right)\right| \\
& \leqq O(1) E \exp \left(-\bar{S}_{m, n}(\lambda)\right)(\log n)^{1 / 4}+\left(E \exp \left(-\bar{S}_{m, n}(\lambda)\right)\right. \\
&\left.+E \exp \left(-\bar{S}_{m, n}^{\prime \prime}(\lambda)\right)\right) I_{\left\{\left|n^{-1} \sum_{k=1}^{n} m^{2} I_{\left\{X_{k} \in Z_{m}^{2}\right\}}-1\right| \geqq(\log n)^{-3 / 8}\right\}} \\
& \leqq O(1)(\log n)^{-1 / 4}+\left(E^{1 / 2} \exp \left(-2 \bar{S}_{m, n}(\lambda)\right)+E^{1 / 2} \exp \left(-2 \bar{S}_{m, n}^{\prime \prime}(\lambda)\right)\right) \\
& \times P^{1 / 2}\left(\left|n^{-1} \sum_{k=1}^{n} m^{2} I_{\left\{X_{k} \in Z_{m}^{2}\right\}}-1\right| \geqq(\log n)^{-3 / 8}\right) \\
& \leqq O(1)(\log n)^{-1 / 4}+O(1) E^{1 / 2} \exp \left(-2 \bar{S}_{m, n}(\lambda)\right) \\
&\left.+E^{1 / 2} \exp \left(-2 \bar{S}_{m, n}^{\prime \prime}(\lambda)\right)\right)(\log n)^{-1 / 2} \\
& \leqq O(1)(\log n)^{-1 / 4}+O(1)(\log n)^{-1 / 2+\delta_{n}},
\end{aligned}
$$

which goes to zero as $n \rightarrow \infty$. Using this fact one can easily show that $\left\{v_{m, n, \lambda}\right\}_{n \geqq 1}$ and $\left\{v_{m, n, \lambda}^{\prime \prime}\right\}_{n \geqq 1}$ have the same asymptotic behaviour for each $\lambda \in[0, \infty)$, provided $\lim \sup _{n \rightarrow \infty} n^{-1} m^{2}(\log n)^{3} / \log \log n=0$.

(ii) Let

$$
\bar{S}_{m, n}^{\prime}(\lambda)=\lambda S_{m, n}-2 \lambda \beta+4 \lambda^{2} n^{-1} \gamma
$$

If $\lim \sup _{n \rightarrow \infty} n^{-1} m^{2}(\log n)^{3}<\infty$, by Lemma 5.2 we know that there is a constant $c_{10} \in(0, \infty)$ such that

$$
E \exp \left(-\bar{S}_{m, n}^{\prime}(\lambda)\right) \geqq \exp \left(-E \bar{S}_{m, n}^{\prime}\right) \geqq c_{10}
$$

In this case, by Proposition 6.1 and Lemma 5.1 we know that $E \exp \left(-\bar{S}_{m, n}^{\prime}(\lambda)\right)$ is less than

$$
E^{1 / 2} \exp \left(-\bar{J}_{1, n}^{m, 4 \lambda}\right) E^{1 / 4} \exp \left(-I_{1, n}^{m, 4 \lambda}\right) E^{1 / 4} \exp \left(8 \lambda^{2} n^{-2} m^{2} \gamma \sum_{k=1}^{n} I_{\left\{X_{k} \in Z_{m}^{2}\right\}}\right) \leqq c_{11}
$$


for some constant $c_{11} \in(0, \infty)$. Let $\varepsilon_{n}=n^{-1} m_{n}^{2}(\log n)^{3}$, and

$$
\eta_{1}(n)=\lambda^{2} n^{-2} \gamma m^{2} \sum_{i=1}^{n} I_{\left\{X_{i} \in Z_{m}^{2}\right\}}, \quad \eta_{2}(n)=\lambda^{2} n^{-1} \gamma .
$$

Again by Lemma 5.2 we can show that

$$
\begin{aligned}
&\left|E \exp \left(-\bar{S}_{m, n}^{\prime}(\lambda)\right)-E \exp \left(-\bar{S}_{m, n}(\lambda)\right)\right| \\
& \leqq E \exp \left(-\bar{S}_{m, n}(\lambda)\right)\left|1-\exp \left(-2 \xi_{1}+2 \xi_{2}+4 \eta_{1}(n)-4 \eta_{2}(n)\right)\right| \\
& \leqq O(1) E \exp \left(-\bar{S}_{m, n}(\lambda)\right) \varepsilon^{1 / 4}+\left(E \exp \left(-\bar{S}_{m, n}(\lambda)\right)\right. \\
&\left.+E \exp \left(-\bar{S}_{m, n}^{\prime}(\lambda)\right)\right) I_{\left\{\left|n^{-1} \sum_{k=1}^{n} m^{2} I_{\left\{X_{k} \in Z_{M}^{2}\right\}}-1\right| \geqq(\log n)^{-1} \varepsilon^{1 / 4}\right\}} \\
& \leqq O(1) \varepsilon_{n}^{1 / 4}+O(1)\left((\log n)^{2} \varepsilon_{n}^{-1 / 2} E\left(n^{-1} \sum_{k=1}^{n} m^{2} I_{\left\{X_{k} \in Z_{m}^{2}\right\}}-1\right)^{2}\right)^{1 / 2} \\
& \leqq O(1) \varepsilon_{n}^{1 / 4},
\end{aligned}
$$

which goes to zero as $n \rightarrow \infty$, if $\lim _{n \rightarrow \infty} \varepsilon_{n}=0$. Using the above estimate and [5, Theorem 1.3] one can show that

$$
v_{m, n, \lambda}^{\prime} \stackrel{\mathscr{D}}{\rightarrow} v_{\lambda}, \quad n \rightarrow \infty
$$

and so

$$
v_{m, n, \lambda} \stackrel{\mathscr{D}}{\rightarrow} v_{\lambda}, \quad n \rightarrow \infty
$$

By (i) we also know that

$$
v_{m, n, \lambda}^{\prime \prime} \stackrel{\mathscr{D}}{\rightarrow} v_{\lambda}, \quad n \rightarrow \infty
$$

which proves the desired result.

Acknowledgements. We are grateful to Professors E. Bolthausen, G.F. Lawler, M. Röckner and G. Slade for their interest and helpful discussions.

\section{Appendix}

The $\phi_{d}^{4}$-field theory (see e.g. [1,3,17 and 18]) is formally described by the following measure:

$$
\prod_{x \in R^{d}} d \phi(x) \exp \left(-\int_{R^{d}}\left(\lambda_{1}|\nabla \phi(x)|^{2}+\lambda_{2} \phi^{2}(x)+\lambda_{3} \phi^{4}(x)\right) d x\right) .
$$

To give a sense to the above formal measure, it is natural to consider its lattice approximations. Let $a Z^{d}=\left\{a x: x \in Z^{d}\right\}$. Heuristically, the quantities $\int|\nabla \phi(x)|^{2} d x$, $\int \phi^{2}(x) d x$ and $\int \phi^{4}(x) d x$ can be approximated respectively by $\Sigma_{|x-y|=a, x, y \in a Z^{d}} a^{d-2}$ $\left(\phi_{x}-\phi_{y}\right)^{2}, a^{d} \Sigma_{x \in a Z^{d}} \phi_{x}^{2}$ and $a^{d} \Sigma_{x \in a Z^{d}} \phi_{x}^{4}$. Thus, one can use the following 
probability measure to approximate (A.1):

$$
\begin{aligned}
N_{a}^{-1} \prod_{x \in a Z^{d}} d \phi_{x} \exp \left(2 \lambda_{1} \sum_{|x-y|=a, x, y \in a Z^{d}} a^{d-2} \phi_{x} \phi_{y}\right. \\
\left.\quad-\left(\lambda_{2} a^{d}+4 d \lambda_{1} a^{d-2}\right) \sum_{x \in a Z^{d}} \phi_{x}^{2}-\lambda_{3} a^{d} \sum_{x \in a Z^{d}} \phi_{x}^{4}\right)
\end{aligned}
$$

where $N_{a}$ is the normalization constant. Let $a^{\prime}=a^{\prime}(a)$ satisfy $\lim _{a \rightarrow 0^{+}} a^{\prime}(a)=0$. For convenience, we assume $a^{\prime} \geqq a$ and $a^{\prime} Z^{d} \subset a Z^{d}$. It is clear that $\int \phi^{4}(x) d x$ can also be approximated by $a^{\prime d} \Sigma_{x \in a^{\prime} Z^{d}} \phi_{x}^{4}$. Thus, it is reasonable to guess that (A.1) can also be approximated by the following probability measure $v_{a, a^{\prime}}$ :

$$
\begin{aligned}
N_{a, a^{\prime}}^{-1} \prod_{x \in a Z^{d}} d \phi_{x} \exp \left(2 \lambda_{1} \sum_{|x-y|=a, x, y \in a Z^{d}} a^{d-2} \phi_{x} \phi_{y}\right. \\
\left.\quad-\left(\lambda_{2} a^{d}+4 d \lambda_{1} a^{d-2}\right) \sum_{x \in a Z^{d}} \phi_{x}^{2}-\lambda_{3} a^{\prime d} \sum_{x \in a^{\prime} Z^{d}} \phi_{x}^{4}\right),
\end{aligned}
$$

where $N_{a, a^{\prime}}$ is the normalization constant. This can be thought of as a discrete $\phi^{4}$ field theory associated to a new cutoff $a^{\prime}$. It is interesting to find a condition on $a^{\prime}(a)$ under which the probability measures $\left\{v_{a, a}\right\}$ and $\left\{v_{a, a^{\prime}}\right\}$ have different limits (if they exist).

We now consider the correlation function of $v_{a, a^{\prime}}$. Following [15] (see also [3 and 12]), we first give a random walk representation for this system. For simplicity, we assume $\lambda_{1}=1$. Let

$$
a_{x y}(a)= \begin{cases}4 a^{d-2}, & |x-y|=a \\ -\left(8 d a^{d-2}+2 \lambda_{2} a^{d}\right), & x=y \\ 0, & \text { otherwise }\end{cases}
$$

Then, there is a continuous time process $\left\{X_{t}\right\}$ on $a Z^{d}$ with the local characteristics $\left\{a_{x y}(a)\right\}$. One can construct a discrete time Markov chain $\left\{Y_{n}\right\}$ with the one-step transition probabilities:

$$
\pi(x, y)= \begin{cases}a_{x y}(a) / a_{x}(a), & |x-y|=a, \\ 1-\sum_{|x-y|=a} \frac{a_{x y}(a)}{a_{x}(a)}, & x \in a Z^{d}, y=\partial, \\ 1, & x=\partial, y=\partial, \\ 0, & \text { otherwise }\end{cases}
$$

where $a_{x}(a)=-a_{x x}(a)$, and $\partial$ is a "cemetery" which is not included in $a Z^{d}$. Thus, the sample path of $\left\{X_{t}\right\}$ can be described by a diagram:

$$
Y_{0} \stackrel{\sigma_{0}}{\rightarrow} Y_{1} \stackrel{\sigma_{1}}{\rightarrow} \cdots Y_{n} \stackrel{\sigma_{n}}{\rightarrow} Y_{\eta} \stackrel{\sigma_{\eta}}{\rightarrow} \partial
$$

where $Y_{0}$ and $Y_{\eta}$ are respectively the initial and final states of $\left\{X_{t}\right\}$. Let $\zeta$ be the time at which $\left\{X_{t}\right\}$ jumps from the final state $Y_{\eta}$ to $\partial$. Let

$$
\tau_{z}=\int_{0}^{\zeta} I_{\left\{X_{t}=z\right\}} d t
$$

and

$$
P_{x y}^{(n)}(C)=Q_{x}\left(C \mid Y_{n}=y, \eta=n\right)
$$


where $\left\{Q_{x}\right\}$ is the probability law of $\left\{X_{t}\right\}$. Let

$$
H(\phi)=-\frac{1}{2} \sum_{|x-y|=a} 4 a^{d-2} \phi_{x} \phi_{y}+\frac{1}{2}\left(2 \lambda_{2} a^{d}+8 d a^{d-2}\right) \sum_{x} \phi_{x}^{2},
$$

and set, for $F$ say bounded and continuous on $R$,

$$
\langle F\rangle=\frac{\int e^{-H(\phi)} F(\phi) d \phi}{\int e^{-H(\phi)} d \phi} .
$$

(This is a heuristic notation for the expectation of $F$ with respect to the well defined centered Gaussian measure of covariance given by the operator $a^{-1}$ in $l^{2}\left(Z^{d}\right)$ ). Using the random walk representation (see e.g. $[3,12,15,17])$, one can obtain that

$$
\left\langle\phi_{x} \phi_{y}\right\rangle_{v_{a, a^{\prime}}}=\sum_{n=0}^{\infty} Q_{x}\left(Y_{n}=y\right) E_{x y}^{(n)} \frac{\left\langle\exp \left(-\lambda_{3} a^{\prime d} \sum_{x \in a^{\prime} Z^{d}}\left(\phi_{x}^{2} / 2+\tau_{x}\right)^{2}\right)\right\rangle}{\left\langle\exp \left(-\lambda_{3} a^{\prime d} \sum_{x \in a^{\prime} Z^{d}}\left(\phi_{x}^{2} / 2\right)^{2}\right)\right\rangle}
$$

where $E_{x y}^{(n)}$ is the expectation with respect to $P_{x y}^{(n)}$. Let us now consider the "zerocomponents $\phi^{4}$ - field" (for its definition, we refer to [10 or 17]). In this case, we have

$$
\begin{aligned}
\left\langle\phi_{x} \phi_{y}\right\rangle_{v_{a, a^{\prime}}} & =\sum_{n=0}^{\infty} Q_{x}\left(Y_{n}=y\right) E_{x y}^{(n)} \exp \left(-\lambda_{3} a^{\prime d} \sum_{x \in a^{\prime} Z^{d}} \tau_{x}^{2}\right) \\
& =\sum_{n=0}^{\infty} Q_{x}\left(Y_{n}=y\right) E_{x y}^{(n)} \exp \left(-\lambda_{3} a^{\prime d} \sum_{x \in a^{\prime} Z^{d}} \int_{0}^{\zeta} \int_{0}^{\zeta} I_{\left\{X_{t}=X_{s}=x\right\}} d t d s\right) .
\end{aligned}
$$

In this formula, the restricted intersection local time (i.e. the random variable $\left.a^{\prime d} \Sigma_{x \in a^{\prime} Z^{d}} \int_{0}^{\zeta} \int_{0}^{\zeta} I_{\left\{X_{t}=X_{s}=x\right\}} d t d s\right)$ has appeared. This is one reason for our interests in discrete Edwards models which are defined in terms of the restricted intersection local times of the random walk. Based on Theorem 2.1 and Theorem 2.2 given in the present paper, we hope to be able to show that the limit of $\left\{v_{a, a^{\prime}}\right\}$ is the same as that of $\left\{v_{a, a}\right\}$ for $d=2$ if $\lim _{a \rightarrow 0^{+}} a^{\prime}|\log a|^{3}=0$ and that the limit of $\left\{v_{a, a^{\prime}}\right\}$ is different from that of $\left\{v_{a, a}\right\}$ for $d=2$ if $a^{\prime}$ is large enough compared to $a$ (e.g. $\lim _{a \rightarrow 0^{+}} a^{\prime}|\log a|^{3}=\infty$ ). We also remark that using results of [15 and 3] (inspired by [28]) expectation with respect to the $\phi_{d}^{4}$ - model measure itself can be expressed in terms of intersection local time. Also in this case then our results on $\left\{v_{a, a^{\prime}}\right\}$ are relevant.

Note added in proof: In recent work (S. Albererio, X.Y. Zhou, A new lattice approximation for the $\varphi_{2}^{4}$-quantum fields, Bochum Preprint '95) we obtained a similar result about $v_{a, a^{\prime}}=v_{a, a}$ in Appendix for the $\varphi_{2}^{4}$ model, under the condition $\lim _{a \rightarrow 0^{+}} a^{\prime}|\log a|^{2}<\infty$. We expect that these measures are different if the latter limit is $\infty$.

\section{References}

1. Aizenman, M.: Geometric analysis of $\phi_{4}^{4}$ fields and Ising models, Parts I and II. Commun. Math. Phys. 86, 1-48 (1982)

2. Albeverio, S., Bolthausen, E., Zhou, X.Y.: On the discrete Edwards model in three dimensions. Preprint (1995) 
3. Albeverio, S., Fenstad, J.E., H $\phi$ egh-Krohn, R., Lindstr $\phi$ m, T.: Nonstandard Methods in Stochastic Analysis and Mathematical Physics. New York: Academic Press, 1986

4. Albeverio, S., Zhou, X.Y.: A modified Domb-Joyce Model in four dimensions. Bochum Preprint (1994) submitted

5. Albeverio, S., Zhou, X.Y.: Renormalization results for some restricted intersection local times. Bochum Preprint (1995) submitted

6. Aragão de Carvalho, C., Caracciolo, S., Fröhlich, J.: Polymers and $g \phi^{4}$ theory in four dimensions. Nucl. Phys. B215, 209-248 (1983)

7. Bolthausen, E.: On the construction of the three dimensional polymer measure. Prob. Th. Rel. Fields 97, 81-101 (1993)

8. Bolthausen, E., Schmock, U.: On self-attracting random walks, pp. 23-44 in: Stochastic Analysis. M.C. Cranston, M.A. Pinsky (eds.) Proc. Symp. Pure Math. 57, Amer. Math. Soc., Providence, 1995

9. Bolthausen, E., Schmock, U.: On self-attracting random walks in arbitrary dimensions. Preprint (1994)

10. Bovier, A., Felder, G., Fröhlich, J.: On the critical properties of the Edwards and self-avoiding walk model of polymer chains. Nucl. Phys. B230, 119-147 (1984)

11. Brydges, D., Evans, S.N., Imbrie, J.Z.: Self-avoiding walk on a hierarchical lattice in four dimensions. Ann. Probab. 20, 82-124 (1992)

12. Brydges, D., Fröhlich, J., Spencer, T.: The random walk representation of classical spin systems and correlation inequalities. Commun. Math. Phys. 83, 123-150 (1982)

13. Brydges, D., Fröhlich, J., Sokal, A.D.: A new proof of the existence and nontriviality of the continuum $\phi_{2}^{4}$ and $\phi_{3}^{4}$ quantum field theories. Commun. Math. Phys. 91, 141-186 (1983)

14. Brydges, D., Slade, G.: The diffusive phase of a model of self-intersecting walks. Preprint (1994)

15. Dynkin, E.B.: Markov processes as a tool in field theory. J. Funct. Anal. 50, 167-187 (1983)

16. Edwards, S.F.: The statistical mecahnics of polymers with excluded volume. Proc. Phys. Soc. (London) 85, 613-624 (1965)

17. Fernandez, R., Fröhlich, J., Sokal, A.D.: Random Walks, Critical Phenomena, and Triviality in Quantum Field Theory. Berlin, Heidelberg, New York: Springer, 1992

18. Fröhlich, J.: On the triviality of $\lambda \phi_{4}^{4}$ theories and the approach to the critical point in $d \geqq 4$ dimensions. Nucl. Phys. B200, 281-296 (1982)

19. Iagolnitzer, D., Magnen, J.: Polymers in a weak random potential in dimension four: Rigorous renormalization group analysis. Commun. Math. Phys. 162, 85-121 (1994)

20. Lawler, G.F.: Intersections of Random Walks. Boston: Birkhäuser, 1991

21. Le Gall, J.F.: Propriétés d'intersection des marches aléatoires, I Convergence vers le temps local d'intersection. Commun. Math. Phys. 104, 471-507 (1986)

22. Le Gall, J.F.: Exponential moments for the renormalized self-intersection local time of planar Brownian motion. To appear in Semin. de Probab. XXVIII, Lect. Notes in Math. (1994)

23. Le Gall, J.F., Rosen, J.: The range of stable random walks. Ann. Probab. 19, 650-705 (1991)

24. Madras, N., Slade, G.: The Self-Avoiding Walk. Boston: Birkhäuser, 1993

25. Rosen, J.: Random walks and intersection local time. Ann. Probab. 18, 959-977 (1990)

26. Stoll, A.: Invariance principles for Brownian intersection local time and polymer measures. Math. Scand. 64, 133-160 (1989)

27. Stroock, D.W., Varadhan, S.R.S.: Multidimensional Diffusion Processes. Berlin, Heidelberg, New York: Springer, 1979

28. Symanzik, K.: Euclidean quantum field theory. In: Local Quantum Field Theory, ed. R. Jost, New York: Academic Press, 1969

29. Westwater, J.: On Edwards' model for long polymer chains. Commun. Math. Phys. 72, 131174 (1980)

30. Westwater, J.: On Edwards' model for long polymer chains III, Borel summability. Commun. Math. Phys. 84, 459-470 (1982)

31. Yor, M.: Renormalization et convergence en loi pour les temps locaux d'intersection du mouvement brownien dans $R^{3}$. Lect. Notes in Math. 1123, 350-365 (1985) 